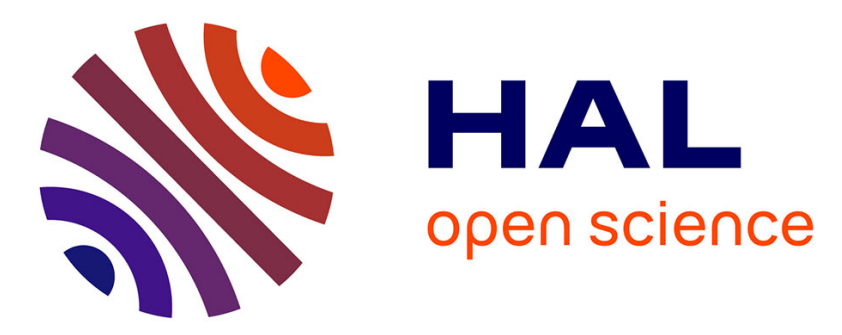

\title{
A robust sensorless output feedback controller of the induction motor drives: New design and experimental validation
}

\author{
Malek Ghanes, J.-P. Barbot, J. Deleon, A. Glumineau
}

\section{- To cite this version:}

Malek Ghanes, J.-P. Barbot, J. Deleon, A. Glumineau. A robust sensorless output feedback controller of the induction motor drives: New design and experimental validation. International Journal of Control, 2010, vol 83, p. 484-497. inria-00450963

\section{HAL Id: inria-00450963 \\ https://hal.inria.fr/inria-00450963}

Submitted on 15 Sep 2010

HAL is a multi-disciplinary open access archive for the deposit and dissemination of scientific research documents, whether they are published or not. The documents may come from teaching and research institutions in France or abroad, or from public or private research centers.
L'archive ouverte pluridisciplinaire HAL, est destinée au dépôt et à la diffusion de documents scientifiques de niveau recherche, publiés ou non, émanant des établissements d'enseignement et de recherche français ou étrangers, des laboratoires publics ou privés. 


\title{
A robust sensorless output feedback controller of the induction motor drives: New design and experimental validation
}

\author{
M. GHANES ${ }^{A 1 *}$, J-B. BARBOT ${ }^{A 1+}$, J. DE LEON ${ }^{A 2} \&$ A. GLUMINEAU ${ }^{A 3}$ \\ ${ }^{A 1}$ Equipe Commande des Systèmes (ECS), ENSEA, 6 Avenue du Ponceau, 95014 Cergy-Pontoise Cedex, France \\ + EP Alien, INRIA \\ ${ }^{A 2}$ U.N.A.L, Department of Electrical Engineering, P.O Box 148-F, 66450, Nuevo Leon, Mexico \\ ${ }^{A 3}$ IRCCyN, UMR CNRS 6597, ECN, BP 92101,1 Rue de la Noe, 44312, Nantes Cedex 3, France \\ (Received 00 Month 200x; In final form 00 Month 200x)
}

\begin{abstract}
In this paper, a sensorless output feedback controller is designed in order to drive the Induction Motor (IM) without the use of flux and speed sensors. Firstly, an observer that uses only the measured stator currents is synthesized to estimate the mechanical variables (speed and load torque) and the magnetic variables (fluxes) by structurally taking into account the unobservability phenomena of the Sensorless $I M(S I M)$ and the parametric uncertainties. Secondly, a current-based field oriented sliding mode control, that uses the flux and the speed estimates given by the former observer is developed so as to steer the estimated speed and flux magnitude to the desired references. Since the observer error dynamic is independent from the known input control and depends on the $I M$ parametric uncertainties, a kind of separation principle is introduced to guarantee the practical stability of the whole closed-loop system "observer -controller" ("O-C") according to observability and unobservability time variation. A significant benchmark taking into account the unobservability phenomena of the SIM is presented to show the performances of the whole control scheme against experimental set-up.
\end{abstract}

\section{Introduction}

High performance electrical drives based on the use of the $I M$ can be implemented by means of speed/flux controllers which rely on field orientation concepts 'Blaschke et al. (1972)'. Generally speaking, a field orientation algorithm is an output feedback controller based on currents and rotor speed/position measurements. In such controllers, a shaft encoder or a resolver is usually used to measure the motor speed. Meanwhile, the presence of this sensor increases the cost and the complexity of the drive system and reduces the reliability of the overall system. Recently, considerable research efforts are focused on the SIM control problem, see 'Holtz et al. (2006)' for an exhaustive overview of IM sensorless control methods. The aim of sensorless techniques is to provide methods of estimating the mechanical speed by using only electrical applied voltages and line currents, as close as possible to the sensor case. Because the load torque is in general unknown, the resistances are temperature varying, the rotor flux and speed are not measurable, several theoretical and practical solutions have been proposed in the open literature. For instance, a speed estimation method is proposed in 'Tajima et al. (1993)' and incorporated in a field-orientation control scheme. In 'Kubota et al. (1994)' an algorithm for simultaneous estimation of motor speed and rotor resistance is proposed. In 'Peresada et al. (1999)', a backstepping output feedback controller based on an indirect field-oriented control is presented. Moreover, estimations of load torque and angle of rotor flux are obtained. By using the estimations of load torque and angle of rotor flux, the controller provides a global asymptotic tracking of smooth speed and flux reference trajectories. In addition, a rotor speed and rotor flux observer which is adaptive with respect to rotor resistance is presented in 'Lin et al. (2000)'. This observer is combined with a state feedback controller which is adaptive with respect to the load torque. In 'Feemster et al. (2001)' a semi-global exponential rotor velocity and flux tracking algorithm is proved assuming the machine parameters are well known and the rotor flux is measured. In 'Marino et al. (2005)' a sensorless controller based on a speed/flux observer is

\footnotetext{
${ }^{*}$ Corresponding author. Email: Malek.Ghanes@ensea.fr
} 
designed under the assumptions of unknown rotor/stator fluxes but with known and smooth load torque. This controller guarantees the local exponential rotor flux tracking with explicit computable attraction domain. Other results dealing with the SIM control problem without load torque knowledge can be found in 'Marino et al. (2007)'.

On the other hand, removing the speed sensors affects the $I M$ observability properties. Important contributions in this direction have been reported in 'Canudas de Wit et al. (2000)', 'Ibarra-Rojas et al. (2004)' and 'Ghanes et al. (2006)', where under some operating conditions (low speed) the IM is not observable. Furthermore, strategies based on IM spatial saliency methods with fundamental excitation and high frequency signal injection 'Holtz et al. (2006)', extended Kalman filter techniques 'Zein et al. (2000)' and adaptive system approaches 'Montanari et al. (2006)' have been studied.

The first main contribution of this paper is to design an interconnected observer for the SIM by a structural consideration of the unobservability phenomena ${ }^{1}$. It is also shown that this observer enables to estimate the speed, the load torque and the magnetic variables by using only stator current measurements according to the observability and unobservability time variation.

Secondly, by using the estimated $I M$ flux and speed given by the observer, a sensorless control scheme is designed. This later is based on a combination of field oriented control (FOC) methodology and robust sliding mode technique. Due to parametric uncertainties of the $I M$ and following partially a "separation principle" introduced in 'Atassi et al. (1999)' and 'Jankovic et al. (1997)', a practical stability of the closed loop system "O-C" is achieved according to the ratio between the unobservable and observable time intervals.

Furthermore, a Sensorless Control Benchmark is designed in order to test and to evaluate the performance of sensorless controllers, particularly when the $I M$ remains in the unobservable conditions (very low speed: zero frequency).

This paper is organized as follows. In section 2, the $I M$ model is reminded. In section 3 , sufficient conditions to ensure the observer convergence by taking into account the SIM unobservability phenomena are given. To achieve the tracking control objective, a sensorless feedback controller combining the FOC and Sliding Mode techniques is designed in Section 4. In Section 5, the closed loop stability analysis using the proposed "O-C" scheme is presented. In Section 6, the benchmark and experimental results showing the efficiency of the proposed control methodology are given ${ }^{2}$. Finally, some conclusions are drawn.

\section{Observer design under unobservability phenomena of $I M$}

\section{$2.1 \quad$ IM Model}

In the $(\alpha, \beta)$ fixed reference frame, the dynamics of the $I M$ reads

$$
\left(\begin{array}{c}
\dot{\phi}_{r \alpha} \\
\dot{\phi}_{r \beta} \\
\dot{i}_{s \alpha} \\
\dot{i}_{s \beta} \\
\dot{\Omega}_{r}
\end{array}\right)=\left(\begin{array}{c}
-a \phi_{r \alpha}-p \Omega_{r} \phi_{r \beta}+a M_{s r} i_{s \alpha} \\
-a \phi_{r \beta}+p \Omega_{r} \phi_{r \alpha}+a M_{s r} i_{s \beta} \\
b\left(a \phi_{r \alpha}+p \Omega_{r} \phi_{r \beta}\right)-\gamma i_{s \alpha} \\
b\left(a \phi_{r \beta}-p \Omega_{r} \phi_{r \alpha}\right)-\gamma i_{s \beta} \\
m\left(\phi_{r \alpha} i_{s \beta}-\phi_{r \beta} i_{s \alpha}\right)-c \Omega_{r}
\end{array}\right)+\left(\begin{array}{ccc}
0 & 0 & 0 \\
0 & 0 & 0 \\
m_{1} & 0 & 0 \\
0 & m_{1} & 0 \\
0 & 0 & -\frac{1}{J}
\end{array}\right)\left(\begin{array}{c}
u_{s \alpha} \\
u_{s \beta} \\
T_{l}
\end{array}\right)
$$

where $i_{s \alpha}, i_{s \beta}, \phi_{r \alpha}, \phi_{r \beta}, u_{s \alpha}, u_{s \beta}, \Omega_{r}, T_{l}$ respectively denote the stator currents, the rotor fluxes, the stator input voltages, the angular rotor speed and the load torque. While, the subscripts $s$ and $r$ refer to the stator and rotor. The parameters $a, b, c, \gamma, \Upsilon, m$ and $m_{1}$ are defined by $a=\left(R_{r} / L_{r}\right), b=\left(M_{s r} / \Upsilon L_{s} L_{r}\right)$,

\footnotetext{
$1_{\text {see section } 2.2 \text { for more details }}$

${ }^{2}$ The proposed control algorithm is experimentally tested and validated using this Benchmark on an experimental set-up located at IRCCyN laboratory of Nantes, France
} 
$c=\left(f_{v} / J\right), \gamma=\left(\frac{L_{r}^{2} R_{s}+M_{s r}^{2} R_{r}}{\Upsilon L_{s} L_{r}^{2}}\right), \Upsilon=\left(1-\left(M_{s r}^{2} / L_{s} L_{r}\right)\right), m=\left(p M_{s r} / J L_{r}\right), m_{1}=\left(1 / \Upsilon L_{s}\right)$. In which $R_{s}$ and $R_{r}$ are the resistances, $L_{s}$ and $L_{r}$ are the self-inductances, $M_{s r}$ is the mutual inductance between the stator and rotor windings, $p$ is the number of pole-pairs, $J$ is the inertia of the system (motor and load) and $f_{v}$ is the viscous damping coefficient. The control inputs are the stator voltages. Only stator currents and stator voltages are measured. Furthermore, an operating domain $\mathcal{D}$ is defined by:

\section{Definition 2.1 : Operation Domain $\mathcal{D}$}

$\Phi_{r \alpha}{ }^{\max }, \Phi_{r \beta}{ }^{\max }, I_{s \beta}{ }^{\max }, I_{s \beta}{ }^{\max }, \Omega_{r}{ }^{\max }$ and $T_{l}^{\max }$ are respectively the actual maximum values for the fluxes, currents, speed and load torque such that $\left|\phi_{r \alpha}\right| \leq \Phi_{r \alpha}{ }^{\max },\left|\phi_{r \beta}\right| \leq \Phi_{r \beta}{ }^{\max },\left|i_{s \alpha}\right| \leq I_{s \alpha}{ }^{\max },\left|i_{s \beta}\right| \leq$ $I_{s \beta}{ }^{\max },\left|\Omega_{r}\right| \leq \Omega_{r}^{\max },\left|T_{l}\right| \leq T_{l}^{\text {max }}$. In the following, the maximum values of parameter variations will be also included for the stability analysis.

\subsection{Quick Recall on the Observability Phenomena of SIM}

The observability phenomena of induction motor has been studied by several authors (see for instance Canudas de Wit et al. (2000), Ibarra-Rojas et al. (2004)). Following the ideas of these works, we have presented in Ghanes et al. (2006) some cases under which the induction motor is observable and unobservable. The problem was to characterize the condition under which the state $x$ of the sensorless induction motor can be observed from measures (currents). The result is that the induction motor observability cannot be established in the particular case when the fluxes $\phi_{r \alpha}, \phi_{r \beta}$ and the speed $\Omega_{r}$ are constant even if we use the higher derivatives of currents. This is a sufficient condition for lost of observability. This operating case match to the following physically interpretation :

1) when the fluxes are constant $\left(\dot{\phi}_{r \beta}=\dot{\phi}_{r \beta}=0\right)$, or equivalently, the excitation voltage is zero $\left(\Omega_{s}=0\right)$, it implies that: $p \Omega_{r}+\frac{R_{r} T_{e}}{p \phi_{d}^{2}}=\Omega_{s}=0$ or: $T_{e m}=-K \Omega_{r}$ where $T_{e m}$ is the electromagnetic torque and $K=\frac{p^{2} \phi_{d}^{2}}{R_{r}}$. $2)$ if the speed motor is constant; thus: $T_{e m}=\left(f_{v} \Omega_{r}+T_{l}\right)=-K \Omega_{r}$. This last equation defines the unobservability curve in the map $\left(T_{l}, \Omega_{r}\right)$ with $M=\frac{p^{2} \phi_{d}^{2}}{R_{r}}+f_{v}$ (Figure 1 ).

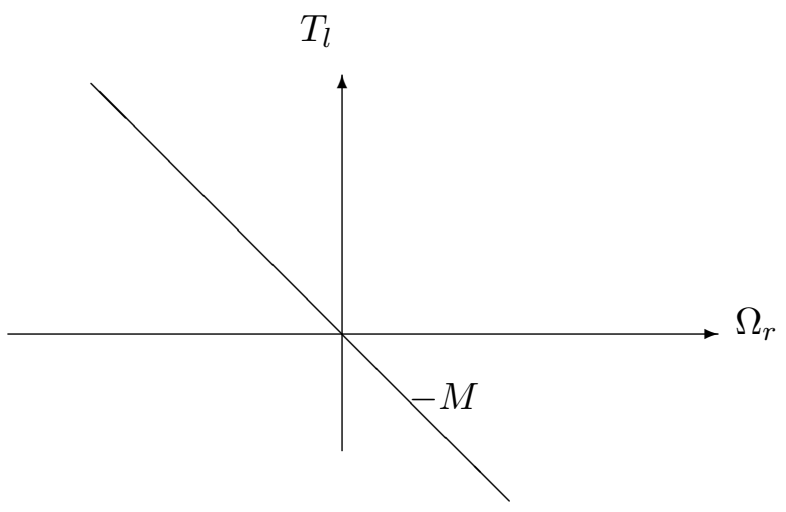

Figure 1. Unobservability curve in the map $\left(T_{l}, \Omega_{r}\right)$.

Obviously, the observability is lost gradually when we approach this unobservability curve.

\subsection{Observer design}

This section is devoted to the SIM observer design. As it is already mentioned, this later takes into account the unobservability phenomena described in section 2.2. The observer design is based on the interconnection of several observers ('Besançon et al. (1998) and 'Besançon et al. (1999)') satisfying suitable properties, in particular the inputs persistency property ('Besançon et al. (1999)', 'Jankovic et al. (1997)'). The main 
idea is to design a set of observers for the whole system from the individual synthesis of an observer for each subsystem. The key issue is that, for each of this observer, the state of the other subsystems is available. In this context, the $I M$ model (1) can be rewritten in the following interconnected extended compact form with parametric uncertainties

$$
\Sigma:\left\{\begin{array}{l}
\Sigma_{1}:\left\{\begin{array}{l}
\dot{X}_{1}=\left[A_{1}\left(X_{2}\right)+\Delta A_{1}\left(X_{2}\right)\right] X_{1}+g_{1}\left(u, y, X_{2}\right)+\Delta g_{1}\left(u, y, X_{2}\right) \\
y_{1}=C_{1} X_{1}
\end{array}\right. \\
\Sigma_{2}:\left\{\begin{array}{l}
\dot{X}_{2}=\left[A_{2}\left(X_{1}\right)+\Delta A_{2}\left(X_{1}\right)\right] X_{2}+\varphi(u, y)+\Delta \varphi(u, y) \\
y_{2}=C_{2} X_{2}
\end{array}\right.
\end{array}\right.
$$

where $X_{1}=\left(i_{s \alpha}, \Omega_{r}, T_{l}\right)^{T}$ and $X_{2}=\left(i_{s \beta}, \phi_{r \alpha}, \phi_{r \beta}\right)^{T}$ are the state of the first and the second subsystem respectively. $u=\left[u_{s \alpha}, u_{s \beta}\right]^{T}$ and $y=\left[i_{s \alpha}, i_{s \beta}\right]^{T}$ are the input and the output vectors of the whole system, and

$$
A_{1}=\left(\begin{array}{ccc}
-\gamma b p \phi_{r \beta} & 0 \\
0 & -c & -\frac{1}{J} \\
0 & 0 & 0
\end{array}\right), A_{2}=\left(\begin{array}{ccc}
-\gamma & -b p \Omega_{r} & a b \\
0 & -a & -p \Omega_{r} \\
0 & p \Omega_{r} & -a
\end{array}\right), g_{1}=\left(\begin{array}{c}
m_{1} u_{s \alpha}+a b \phi_{r \alpha} \\
m \phi_{r \alpha} i_{s \beta}-m \phi_{r \beta} i_{s \alpha} \\
0
\end{array}\right), \varphi=\left(\begin{array}{c}
m_{1} u_{s \beta} \\
a M_{s r} i_{s \alpha} \\
a M_{s r} i_{s \beta}
\end{array}\right)
$$

$C_{1}=C_{2}=\left(\begin{array}{lll}1 & 0 & 0\end{array}\right)$.

$u \in U$ is the set of admissible inputs and $n_{i}$ is the dimension of each subsystem $\left(n_{1}=n_{2}=3\right)$. $T_{l}$ is an unknown load torque which is assumed constant. The terms $\Delta A_{1}\left(X_{2}\right), \Delta A_{2}\left(X_{1}\right), \Delta g_{1}\left(u, y, X_{2}\right)$ and $\Delta \varphi(u, y)$ represent the uncertain terms of $A_{1}\left(X_{2}\right), A_{2}\left(X_{1}\right), g_{1}\left(u, y, X_{2}\right), \varphi(u, y)$ respectively.

Let us now introduce the following property and definition:

Property 2.2 a- Since $A_{1}\left(X_{2}\right)$ and $A_{2}\left(X_{1}\right)$ are linear, they are respectively globally Lipschitz with respect to $X_{2}, X_{1}$.

b- $g_{1}\left(u, y, X_{2}\right)$ is Lipschitz with respect to the flux and uniformly with respect to $(u, y)$ as long as the $I M$ (1) state remains in $\mathcal{D}$.

c- Due to the fact that the matrix $A_{1}$ is Lyapunov stable $(\gamma>0$ and $c>0)$, there exists a positive matrix $S_{1}>0$ such that $A_{1}^{T} S_{1}+S_{1} A_{1}=-Q$ where $Q \geq 0$.

d- Due to the fact that the matrix $A_{2}$ is exponentially stable $(\gamma>0, a>0)$, there exists a positive matrix $S_{2}>0$ such that $A_{2}^{T} S_{2}+S_{2} A_{2}=-I$.

Definition 2.3 Let $\mathbf{D}=\operatorname{det} \mathcal{O}_{J}$, where $\mathcal{O}_{J}=\frac{\partial}{\partial \tilde{X}}(\mathcal{O})$ is the jacobian observability matrix and $\tilde{X}=\left(i_{s \alpha} i_{s \beta} \hat{\phi}_{r \alpha} \hat{\phi}_{r \beta} \hat{\Omega}_{r} \hat{T}_{l}\right)^{T}$. The $I M$ associated observability subspace $\mathcal{O}$ is generated by $\mathcal{O}=\left(i_{s \alpha} i_{s \beta} i_{s \alpha}^{(1)} i_{s \beta}^{(1)} i_{s \alpha}^{(2)} i_{s \beta}^{(2)}\right)^{T}$.

$\mathbf{D}_{\min }$ is the smallest value of $\mathbf{D}$ chosen such that the $I M$ is in the observable area.

Now, from property 2.2, definition 2.3 and taking into account the unobservability phenomena of the SIM described in section 2.2, sufficient conditions are given in the sequel such that system (3) is a practical exponential observer for the whole system (2).

$$
O:\left\{\begin{aligned}
O_{1}:\left\{\begin{array}{l}
\dot{Z}_{1}=A_{1}\left(Z_{2}\right) Z_{1}+g_{1}\left(u, y, Z_{2}\right)+\mathbf{M} S_{1}^{-1} C_{1}^{T}\left(y_{1}-\hat{y}_{1}\right) \\
\dot{S}_{1}=\mathbf{M}\left(-\theta_{1} S_{1}-A_{1}^{T}\left(Z_{2}\right) S_{1}-S_{1} A_{1}\left(Z_{2}\right)+C_{1}^{T} C_{1}\right) \\
\hat{y}_{1}=C_{1} Z_{1}
\end{array}\right. \\
O_{2}:\left\{\begin{array}{l}
\dot{Z}_{2}=A_{2}\left(Z_{1}\right) Z_{2}+\varphi(u, y)+\mathbf{M} S_{2}^{-1} C_{2}^{T}\left(y_{2}-\hat{y}_{2}\right) \\
\dot{S}_{2}=\mathbf{M}\left(-\theta_{2} S_{2}-A_{2}^{T}\left(Z_{1}\right) S_{2}-S_{2} A_{2}\left(Z_{1}\right)+C_{2}^{T} C_{2}\right) \\
\hat{y}_{2}=C_{2} Z_{2}
\end{array}\right. \\
M=1 \text { if }|D|>D_{\text {min }} ; \quad M=\frac{|D|}{D_{\text {min }}} \text { if }|D|<D_{\text {min }} ; \\
M=0 \text { if }|D|=0
\end{aligned}\right.
$$


where $Z_{1}=\left(\hat{i}_{s \alpha}, \hat{\Omega}_{r}, \hat{T}_{l}\right)^{T}, Z_{2}=\left(\hat{i}_{s \beta}, \hat{\phi}_{r \alpha}, \hat{\phi}_{r \beta}\right)^{T}, S_{i}=S_{i}^{T}>0, \mathrm{i}=1,2 . \theta_{1}$ and $\theta_{2}$ are positive constants. Note that $S_{1}^{-1} C_{1}^{T}$ and $S_{2}^{-1} C_{2}^{T}$ are the respective gains of observers $\left(O_{1}\right)$ and $\left(O_{2}\right)$. Furthermore, when the $I M$ is no longer in the observable area, observer (3) can not work any more. Then, a solution based on the $I M$ observability property is introduced by using a soft switch function $\mathbf{M}$ such that observer (3) operates as an estimator.

Remark 1 1. $Z_{2}$ and $Z_{1}$ are considered as inputs for subsystems $\left(O_{1}\right)$ and $\left(O_{2}\right)$, the solutions of $\dot{S}_{1}$ and $\dot{S}_{2}$ are symmetric positive definite matrices (see annexe for the positiveness proof of $S_{i}(t), i=1,2$ ).

2. When the $I M$ is in the observable area, $Z_{2}$ and $Z_{1}$ satisfy the regularly persistence condition.

3. When the $I M$ is in the unobservable area, $Z_{2}$ and $Z_{1}$ do not satisfy the regularly persistence condition and the observer operates as an estimator.

4. It is worth noticing that $\left\|S_{1}\right\|$ and $\left\|S_{2}\right\|$ are bounded when the $I M$ is in the observable area.

\subsection{Observer convergence}

In order to prove the convergence of the proposed observer (3), sufficient conditions are established. Defining the estimation errors

$$
\left\{\begin{array}{l}
\epsilon_{1}=X_{1}-Z_{1} \\
\epsilon_{2}=X_{2}-Z_{2}
\end{array}\right.
$$

According to system (2) and observer (3), their errors dynamics read

$$
\Sigma_{\epsilon}:\left\{\begin{aligned}
\dot{\epsilon_{1}} & =\left[A_{1}\left(Z_{2}\right)-\mathbf{M} S_{1}^{-1} C_{1}^{T} C_{1}\right] \epsilon_{1}+g_{1}\left(u, y, X_{2}\right)+\Delta g_{1}\left(u, y, X_{2}\right)-g_{1}\left(u, y, Z_{2}\right) \\
& +\left[A_{1}\left(X_{2}\right)+\Delta A_{1}\left(X_{2}\right)-A_{1}\left(Z_{2}\right)\right] X_{1} \\
\dot{\epsilon_{2}} & =\left[A_{2}\left(Z_{1}\right)-\mathbf{M} S_{2}^{-1} C_{2}^{T} C_{2}\right] \epsilon_{2}+\Delta \varphi(u, y)+\left[A_{2}\left(X_{1}\right)+\Delta A_{2}\left(X_{1}\right)-A_{2}\left(Z_{1}\right)\right] X_{2} .
\end{aligned}\right.
$$

Proposition 2.4 Consider system (2) with property 2.2. Then, system (3) is a practical exponential observer $^{1}$ for system (2), for $\theta_{1}>0$ and $\theta_{2}>0$ sufficiently large.

Proof Consider the following Lyapunov function candidate

$$
V_{o}=V_{1}+V_{2}
$$

where $V_{1}=\epsilon_{1}^{T} S_{1} \epsilon_{1}$ and $V_{2}=\epsilon_{2}^{T} S_{2} \epsilon_{2}$.

Remark 2 The Lyapunov function candidate (5) is well chosen since $S_{i}(t), i=1,2$ is positive definite (see annexe for more details)

Its time derivative along (4) is

$$
\begin{aligned}
\dot{V}_{o}= & -M \epsilon_{1}^{T} C_{1}^{T} C_{1} \epsilon_{1}-M \theta_{1} \epsilon_{1}^{T} S_{1} \epsilon_{1}+\epsilon_{1}^{T}\left[A_{1}^{T}\left(Z_{2}\right) S_{1}+S_{1} A_{1}\left(Z_{2}\right)\right] \epsilon_{1}(1-M) \\
& +2 \epsilon_{1}^{T} S_{1}\left[A_{1}\left(X_{2}\right)+\Delta A_{1}\left(X_{2}\right)-A_{1}\left(Z_{2}\right)\right] X_{1}+2 \epsilon_{1}^{T} S_{1}\left[g_{1}\left(u, y, X_{2}\right)+\Delta g_{1}\left(u, y, X_{2}\right)-g_{1}\left(u, y, Z_{2}\right]\right. \\
& -M \epsilon_{2}^{T} C_{2}^{T} C_{2} \epsilon_{2}-M \theta_{2} \epsilon_{2}^{T} S_{2} \epsilon_{2}+\epsilon_{2}^{T}\left[A_{2}\left(Z_{1}\right)^{T} S_{2}+S_{2} A_{2}\left(Z_{1}\right)\right] \epsilon_{2}(1-M) \\
& +2 \epsilon_{2}^{T} S_{2}\left[A_{2}\left(X_{1}\right)+\Delta A_{2}\left(X_{1}\right)-A_{2}\left(Z_{1}\right)\right] X_{2}+2 \epsilon_{2}^{T} S_{2} \Delta \varphi(u, y) X_{2} .
\end{aligned}
$$

\footnotetext{
${ }^{1}$ the observer is exponentially convergent to a ball $B_{r}$
} 
By introducing the norm, it follows

$$
\begin{aligned}
\dot{V}_{o} \leq- & M \epsilon_{1}^{T} C_{1}^{T} C_{1} \epsilon_{1}-M \theta_{1} \epsilon_{1}^{T} S_{1} \epsilon_{1}-M \theta_{1} \epsilon_{1}^{T} S_{1} \epsilon_{1}+\epsilon_{1}^{T}\left\{A_{1}^{T}\left(Z_{2}\right) S_{1}+S_{1} A_{1}\left(Z_{2}\right)\right\} \epsilon_{1}(1-M) \\
& +2\left\|\epsilon_{1}\right\|\left\|S_{1}\right\|\left\|\left\{A_{1}\left(X_{2}\right)+\Delta A_{1}\left(X_{2}\right)-A_{1}\left(Z_{2}\right)\right\}\right\|\left\|X_{1}\right\| \\
& +2\left\|\epsilon_{1}\right\|\left\|S_{1}\right\|\left\|\left\{g_{1}\left(u, y, X_{2}\right)+\Delta g_{1}\left(u, y, X_{2}\right)-g_{1}\left(u, y, Z_{2}\right)\right\}\right\| \\
& -M \epsilon_{2}^{T} C_{2}^{T} C_{2} \epsilon_{2}-M \theta_{2} \epsilon_{2}^{T} S_{2} \epsilon_{2}+\epsilon_{2}^{T}\left\{A_{2}^{T}\left(Z_{1}\right) S_{2}+S_{2} A_{2}\left(Z_{1}\right)\right\} \epsilon_{2}(1-M) \\
& +2\left\|\epsilon_{2}\right\|\left\|S_{2}\right\|\left\|\left\{A_{2}\left(X_{1}\right)+\Delta A_{2}\left(X_{1}\right)-A_{2}\left(Z_{1}\right)\right\}\right\|\left\|X_{2}\right\|+2\left\|\epsilon_{2}\right\|\left\|S_{2}\right\|\|\{\Delta \varphi(u, y)\}\|\left\|X_{2}\right\| .
\end{aligned}
$$

From property 2.2-a-b, the set inequalities hold

$$
\left\{\begin{array}{l}
\left\|S_{1}\right\| \leq k_{1} ;\left\|\left\{A_{1}\left(X_{2}\right)-A_{1}\left(Z_{2}\right)\right\}\right\| \leq k_{2}\left\|\epsilon_{2}\right\| ;\left\|X_{1}\right\| \leq k_{3} \\
\left\|\left\{g_{1}\left(u, y, X_{2}\right)-g_{1}\left(u, y, Z_{2}\right)\right\}\right\| \leq k_{4}\left\|\epsilon_{2}\right\| ;\left\|S_{2}\right\| \leq k_{5} ; \\
\left\|\left\{A_{2}\left(X_{1}\right)-A_{2}\left(Z_{1}\right)\right\}\right\| \leq k_{6}\left\|\epsilon_{1}\right\| ;\left\|X_{2}\right\| \leq k_{7}
\end{array}\right.
$$

Using the above inequalities and because the fact pointed in property 2.2-c and 2.2-d, (6) becomes

$$
\begin{aligned}
\dot{V}_{o} \leq & -M \epsilon_{1}^{T} C_{1}^{T} C_{1} \epsilon_{1}-M \theta_{1} \epsilon_{1}^{T} S_{1} \epsilon_{1}-\epsilon_{1}^{T} Q \epsilon_{1}(1-M)+2 \mu_{1}\left\|\epsilon_{1}\right\|\left\|\epsilon_{2}\right\|+2 \mu_{2}\left\|\epsilon_{1}\right\|\left\|\epsilon_{2}\right\| \\
& -\epsilon_{2}^{T} \epsilon_{2}(1-M)+\mu_{4}\left\|\epsilon_{1}\right\|-M \epsilon_{2}^{T} C_{2}^{T} C_{2} \epsilon_{2}-M \theta_{2} \epsilon_{2}^{T} S_{2} \epsilon_{2}+2 \mu_{3}\left\|\epsilon_{2}\right\|\left\|\epsilon_{1}\right\|+\mu_{5}\left\|\epsilon_{2}\right\|
\end{aligned}
$$

where $\mu_{1}=k_{1} k_{2} k_{3}, \mu_{2}=k_{1} k_{4}, \mu_{3}=k_{5} k_{6} k_{7}, \mu_{4}=2\left(k_{1} k_{3} \rho_{1}+k_{1} \rho_{3}\right), \mu_{5}=2\left(k_{5} k_{7} \rho_{2}+k_{5} \rho_{4}\right)$.

The parameters $k_{j}, j=1, \ldots, 8$ and $\rho_{i}, i=1, \ldots, 4$ are positives constants which are computed by determining the maximal values of $A_{1}, A_{2}, g_{1}, S_{1}, S_{2}, \Delta A_{1}\left(X_{2}\right), \Delta A_{2}\left(X_{1}\right), \Delta g_{1}\left(u, y, X_{2}\right)$ and $\Delta \varphi(u, y)$ in the physical domain $\mathcal{D}$ of $I M$ (see definition 2.1).

Since $0 \leq M \leq 1, Q \geq 0$ and $C_{i}^{T} C_{i} \geq 0, i=1,2,(7)$ follows

$$
\begin{aligned}
\dot{V}_{o} \leq & -M \theta_{1} \epsilon_{1}^{T} S_{1} \epsilon_{1}+2 \mu_{1}\left\|\epsilon_{1}\right\|\left\|\epsilon_{2}\right\|+2 \mu_{2}\left\|\epsilon_{1}\right\|\left\|\epsilon_{2}\right\| \\
& +\mu_{4}\left\|\epsilon_{1}\right\|-M \theta_{2} \epsilon_{2}^{T} S_{2} \epsilon_{2}+2 \mu_{3}\left\|\epsilon_{2}\right\|\left\|\epsilon_{1}\right\|+\mu_{5}\left\|\epsilon_{2}\right\|
\end{aligned}
$$

Using the following inequalities

$$
\left\{\begin{array}{l}
\lambda \min \left(S_{i}\right)\left\|\epsilon_{i}\right\|^{2} \leq\left\|\epsilon_{i}\right\|_{S_{i}}^{2} \leq \lambda \max \left(S_{i}\right)\left\|\epsilon_{i}\right\|^{2} \\
\left\|\epsilon_{i}\right\|_{S_{i}}^{2}=\epsilon_{i}^{T} S_{i} \epsilon_{i}, i=1,2
\end{array}\right.
$$

where $\lambda \min \left(S_{i}\right)$ and $\lambda \max \left(S_{i}\right)$ are respectively the minimal and maximal eigenvalues of $S_{i}$.

By writing (8) in terms of functions $V_{1}$ and $V_{2}$, it follows that

$$
\dot{V}_{o} \leq-M\left(\theta_{1} V_{1}-\theta_{2} V_{2}\right)+2\left(\tilde{\mu}_{1}+\tilde{\mu}_{2}+\tilde{\mu}_{3}\right) \sqrt{V_{1}} \sqrt{V_{2}}+\mu_{4}\left\|\epsilon_{1}\right\|+\mu_{5}\left\|\epsilon_{2}\right\|
$$

where $\tilde{\mu}_{i}=\frac{\mu_{i}}{\sqrt{\lambda_{\min }\left(S_{1}\right)} \sqrt{\lambda_{\min }\left(S_{2}\right)}}, i=1,2,3$.

Next, by using the following inequality $\left.\sqrt{V_{1}} \sqrt{V_{2}} \leq \frac{v}{2} V_{1}+\frac{1}{2 v} V_{2}, \forall v \in\right] 0,1$, one get 


$$
\dot{V}_{o} \leq-\left(\mathbf{M} \theta_{1}-N v\right) V_{1}-\left(\mathbf{M} \theta_{2}-\frac{N}{v}\right) V_{2}+\mu_{4}\left\|\epsilon_{1}\right\|+\mu_{5}\left\|\epsilon_{2}\right\|
$$

where $N=\tilde{\mu}_{1}+\tilde{\mu}_{2}+\tilde{\mu}_{3} . \tilde{\mu}_{i}=\frac{\mu_{i}}{\sqrt{\lambda_{\min }\left(S_{1}\right)} \sqrt{\lambda_{\min }\left(S_{2}\right)}}, i=1,2,3 . \mu_{1}=k_{1} k_{2} k_{3}, \mu_{2}=k_{1} k_{4}, \mu_{3}=k_{5} k_{6} k_{7}$, $\mu_{4}=2\left(k_{1} k_{3} \rho_{1}+k_{1} \rho_{3}\right), \mu_{5}=2\left(k_{5} k_{7} \rho_{2}+k_{5} \rho_{4}\right)$.

Tacking account $\mu=\max \left(\mu_{4}, \mu_{5}\right)$, it follows that:

1) when $\mathbf{M}=1$ (observable conditions), (10) becomes:

$$
\dot{V}_{o}(\mathbf{M}=1) \leq-\delta^{\prime} V_{o}+r
$$

where

$$
\left\{\begin{array}{l}
\delta^{\prime}=\left(\delta-\frac{1}{2 v}\right) \\
r=\mu^{2} \frac{v}{2} \\
\delta=\min \left(\delta_{1}, \delta_{2}\right) \\
\delta_{1}=\left(\theta_{1}-N v\right)>0 \\
\delta_{2}=\left(\theta_{2}-\frac{N}{v}\right)>0
\end{array}\right.
$$

By choosing $\delta>\frac{1}{2 v}$, it implies that the origin of system (4) is practically exponentially stable (more details about practical stability can be found in 'Laskhmikanthan et al. (1990)' and 'Panteley et al. (1998)').

2) when $\mathbf{M}=0$ (unobservable conditions), (10) becomes:

$$
\dot{V}_{o}(\mathbf{M}=0) \leq \tilde{K}^{\prime} V_{o}+r
$$

where $\tilde{K}^{\prime}=\left(\tilde{K}+\frac{1}{2 v}\right)$ and $\tilde{K}=\max \left(N v, \frac{N}{v}\right)$.

By defining the time trajectory as $T=\tau_{o b s}+\tau_{\text {unobs }}$ (see figure 2 ), then

For $\mathbf{M}=1$ :

$$
V_{o}^{0}(\mathbf{M}=1) \leq \exp ^{-\delta^{\prime} \tau_{o b s}} V_{o}^{0}(\mathbf{M}=0)+\frac{1-e^{-\delta^{\prime} \tau_{o b s}}}{\delta^{\prime}} r .
$$

For $\mathbf{M}=0$ :

$$
V_{o}^{1}(\mathbf{M}=0) \leq e^{\tilde{K}^{\prime} \tau_{u n o b s}} V_{o}^{0}(\mathbf{M}=1)+\frac{e^{\tilde{K}^{\prime} \tau_{u n o b s}}-1}{\tilde{K}^{\prime}} r .
$$

Now, define the difference between the Lyapunov functions $V_{o}^{1}(\mathbf{M}=0)$ and $V_{o}^{0}(\mathbf{M}=0)$ (as introduced in Balluchi et al. (2003)) in order to study the sign of the variation of the Lyapunov function $V_{o}$ at all $T^{1}$ :

${ }^{1}$ cycle of observable and unobservable conditions 


$$
\begin{aligned}
\Delta V_{o} \leq V_{o}^{1}(\mathbf{M}=0)-V_{o}^{0}(\mathbf{M}=0): & =\left(e^{\tilde{K}^{\prime} \tau_{u n o b s}-\delta^{\prime} \tau_{o b s}}-1\right) V_{o}^{0}(\mathbf{M}=0) \\
& +\left[e^{\tilde{K}^{\prime} \tau_{\text {unobs }}}\left(\frac{1-e^{-\delta^{\prime} \tau_{o b s}}}{\delta^{\prime}}\right)+\frac{e^{\tilde{K}^{\prime} \tau_{u n o b s}}-1}{\tilde{K}^{\prime}}\right] r .
\end{aligned}
$$

It is obvious that only practical stability is obtained for

$$
\tilde{K}^{\prime} \tau_{\text {unobs }}-\delta^{\prime} \tau_{\text {obs }}<0
$$

(12) ensures that the Lyapunov function $V_{0}$ converges into the interval $\left[0, V_{0}^{\max }\right]$ where

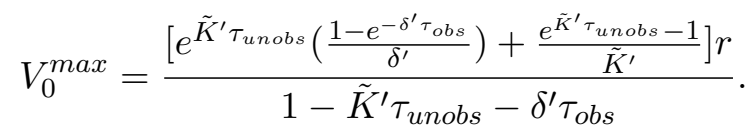

If $r=0$, then exponential convergence to zero is obtained.

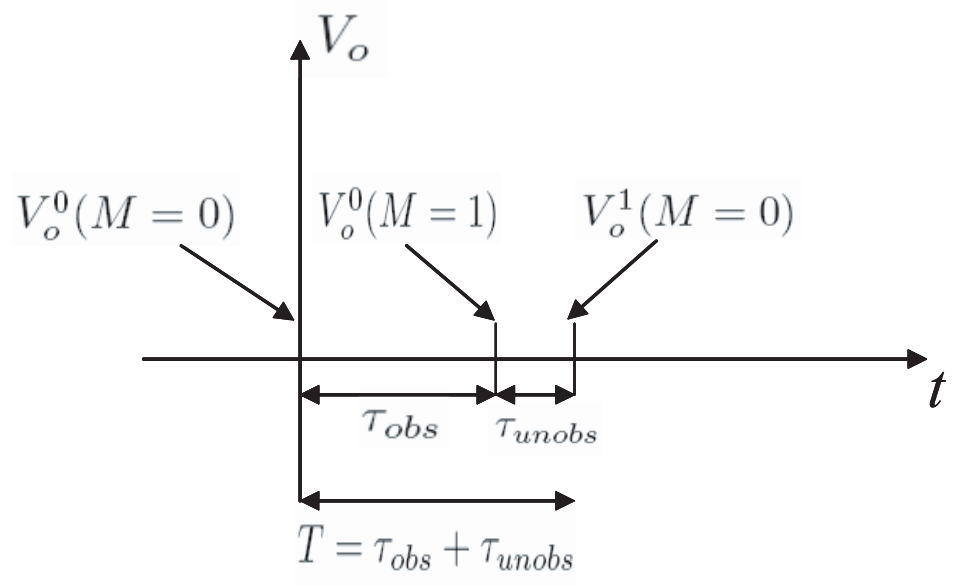

Figure 2. Lyapunov function $V_{o}$ for $T=\tau_{o b s}+\tau_{\text {unobs }}$.

\section{$3 \quad$ FOC via Sliding Mode Techniques}

In this section, a controller is designed by combining the $\boldsymbol{F} \boldsymbol{O C}$ method ('Blaschke et al. (1972)') with Sliding Mode Control method ( $\boldsymbol{S M C}$, 'Utkin et al. (1992)'). The design procedure is based on the assumption of current-fed $I M$.

Field Oriented Control. In the rotating (d-q) reference frame, the $I M$ dynamic model (1) reads (Chiasson et al. (2005))

$$
\left\{\begin{aligned}
\dot{\Omega}_{r} & =m \phi_{r d} i_{s q}-c \Omega_{r}-\frac{1}{J} T_{l} \\
\dot{\phi_{r d}} & =-a \phi_{r d}+a M_{s r} i_{s d} \\
\dot{\rho} & =p \Omega_{r}+a \frac{M_{s r}}{\phi_{r d}} i_{s q} \\
\dot{i}_{s d} & =-\gamma i_{s d}+a b \phi_{r d}+p \Omega_{r} i_{s q}+a \frac{M_{s r}}{\phi_{r d}} i_{s q}^{2}+m_{1} u_{s d} \\
\dot{i}_{s q} & =-\gamma i_{s q}-b p \Omega_{r} \phi_{r d}-p \Omega_{r} i_{s d}-a \frac{M_{s r}}{\phi_{r d}} i_{s d} i_{s q}+m_{1} u_{s q}
\end{aligned}\right.
$$

where respectively $i_{s d}, i_{s q}$ and $u_{s d}, u_{s q}$ are the stator currents and stator voltages. The electromagnetic 
torque $T_{e m}=\frac{p M_{s r}}{L_{r}} \phi_{r d} i_{s q}$ is proportional to the product of $\phi_{r d}$ and $i_{s q}$. Thus, by holding constant the magnitude of the rotor flux, a linear relationship between $i_{s q}$ and $T_{e m}$ is obtained. In order to cancel the nonlinear dynamics of $i_{s d}$ and $i_{s q}$, the system is forced into current-command mode using high gain feedback (see Chiasson et al. (2005)). More precisely, the following IP current controllers

$$
\left\{\begin{array}{l}
V_{s d}=K i_{v d} \int_{0}^{t}\left(i_{s d}^{*}-i_{s d}\right) d t+K p_{v d}\left(i_{s d}^{*}-i_{s d}\right) \\
V_{s q}=K i_{v q} \int_{0}^{t}\left(i_{s q}^{*}-i_{s q}\right) d t+K p_{v q}\left(i_{s q}^{*}-i_{s q}\right)
\end{array}\right.
$$

are used to force $i_{s d}$ and $i_{s q}$ to track their respective references $i_{s d}^{*}$ and $i_{s q}^{*}$ and produce fast responses when large feedback gains are used. Hence, assuming that $i_{s d}^{*}$ and $i_{s q}^{*}$ as the new inputs, it follows that

$$
\left\{\begin{array}{l}
\dot{\Omega}_{r}=m \phi_{r d} i_{s q}^{*}-c \Omega_{r}-\frac{T_{l}}{J} \\
\dot{\phi_{r d}}=-a \phi_{r d}+a M_{s r} i_{s d}^{*}
\end{array}\right.
$$

In order to solve the flux and speed trajectory tracking problem, the following assumption is introduced.

Assumption 3.1 a- The state initial conditions of the $I M$ are in the physical domain $\mathcal{D}$.

b- The desired trajectories $\left(\phi_{r d}^{*}\right.$ and $\left.\Omega_{r}^{*}\right)$ are in the physical domain $\mathcal{D}$.

c- The actual load torque is assumed to be bounded by a maximal fixed value $\varrho$. This maximal value is chosen in accordance to the realistic torque characteristics of the chosen drive: $\left|T_{l}\right|<\varrho$.

\section{Sliding Mode Control.}

Flux controller design. From (15), consider the following $I M$ flux dynamic equation with uncertainties

$$
\dot{\phi}_{r d}=-a \phi_{r d}+\Delta a \phi_{r d}+\kappa i_{s d}^{*}
$$

where $\kappa=a M_{s r}$ and $\Delta a$ is the uncertainty term of parameter $a$. In order to design a flux sliding mode controller, define the flux tracking error $e_{\phi_{r d}}=\phi_{r d}-\phi_{r d}^{*}$ where $\phi_{r d}^{*}$ is the flux reference. The associated error dynamics is

$$
\dot{e}_{\phi_{r d}}=-a e_{\phi_{r d}}+\kappa i_{s d}^{*}-a \phi_{r d}^{*}-\dot{\phi}_{r d}^{*}+\Delta a \phi_{r d}
$$

From $S M C$ theory, define the $\phi_{r d}$ flux sliding manifold as

$$
\sigma_{\phi_{r d}}=e_{\phi_{r d}}-\left(k_{\phi_{r d}}-a\right) \int_{0}^{t} e_{\phi_{r d}}(\tau) d \tau
$$

The associated Lyapunov function is selected as

$$
V_{\sigma_{\phi_{r d}}}=\frac{1}{2} \sigma_{\phi_{r d}}^{2}
$$

Its time derivative is given by

$$
\dot{V}_{\sigma_{\phi_{r d}}}=\sigma_{\phi_{r d}}\left[\sigma_{\phi_{r d} 1}+\sigma_{\phi_{r d}} i_{s d}^{*}+\Delta a \phi_{r d}\right]
$$

where $\sigma_{\phi_{r d} 1}=k_{\phi_{r d}} e_{\phi_{r d}}+a \phi_{r d}^{*}+\dot{\phi}_{r d}^{*}$ and $\sigma_{\phi_{r d} 2}=\kappa$. 
Therefore, the sliding mode controller follows

$$
i_{s d}^{*}=i_{s d, e q u}^{*}+i_{s d, n}^{*}
$$

In this equation $i_{s d, e q u}^{*}=-\frac{\sigma_{\phi_{r d} 1}}{\sigma_{\phi_{r d}} 2}-l_{\phi_{r d}} \sigma_{\phi_{r d}}$ is the equivalent control and $i_{s d, n}^{*}=-\frac{u_{d n}}{\sigma_{\phi_{r d} d^{2}}}$ is the discontinuous control where $u_{d n}=\eta_{\phi_{r d}} \operatorname{sgn}\left(\sigma_{\phi_{r d}}\right)$ with

$$
\operatorname{sgn}\left(\sigma_{\phi_{r d}}\right):\left\{\begin{array}{l}
1 \text { if } \sigma_{\phi_{r d}}>0 \\
-1 \text { if } \sigma_{\phi_{r d}}<0 \\
\in[-1,1] \text { if } \sigma_{\phi_{r d}}=0
\end{array}\right.
$$

Then (18) becomes

$$
\dot{V}_{\sigma_{\phi_{r d}}}=-l_{\phi_{r d}} V_{\sigma_{\phi_{r d}}}+\sigma_{\phi_{r d}}\left[-\eta_{\phi_{r d}} \operatorname{sgn}\left(\sigma_{\phi_{r d}}\right)+\Delta a \phi_{r d}\right]
$$

Choosing $l_{\phi_{r d}}>0$ and $\eta_{\phi_{r d}}>\max \left\{\left\|\Delta a \phi_{r d}\right\|\right\}$ (defined hereafter) it follows that $\dot{V}_{\sigma_{\phi_{r d}}} \leq 0$. As $V_{\sigma_{\phi_{r d}}}$ is contracting and from assumption $(3.1-\mathrm{a}-\mathrm{b})$ then $\max \left\{\phi_{r d}\right\}:=K_{\phi_{r d}}^{\max }$ can not be greater than $\max \left\{\phi_{r d}(0), \phi_{r d}^{*}\right\}+\left|\Delta e_{\phi_{r d}}(0)\right|$. Consequently, $\Delta a \phi_{r d}$ is bounded and can be set as $\eta_{\phi_{r d}}=\Delta a^{\max } K_{\phi_{r d}}^{\max }+b_{\phi}$, with $b_{\phi}$ a small positive constant. Furthermore, all the trajectories reach the sliding manifold $\sigma_{\phi_{r d}}=0$ in a finite time and remain there. Therefore $\sigma_{\phi_{r d}}=0$ and equation (17) becomes

$$
\dot{e}_{\phi_{r d}}=\left(k_{\phi_{r d}}-a\right) e_{\phi_{r d}}
$$

Hence, the flux tracking error $e_{\Omega_{r}}$ exponentially converges to 0 for $\left(k_{\phi_{r d}}-a\right)<0$.

Now, choosing (19) to force $\phi_{r d}$ to track its reference $\phi_{r d}^{*}$ ensures that the flux is properly established in the motor. Hence, after the $I M$ is fluxed $\left(\phi_{r d}=\phi_{r d}^{*}=\right.$ constant), the electromagnetic torque $\left(T_{e m}\right)$ can be rewritten as $T_{e m}=K_{T} i_{s q}^{*}$, where $K_{T}$ is the motor torque constant defined by $K_{T}=\frac{p M_{s r}}{L_{r}} \phi_{r d}$. As a consequence, the linear relationship between the input $i_{s q}^{*}$ and the speed dynamics $\dot{\Omega}_{r}$ is obtained. Then, the speed control is obtained through the input $i_{s q}^{*}$ via a speed controller described below.

Speed controller design. Consider the mechanical equation of (15) including uncertainties

$$
\dot{\Omega}_{r}=-c \Omega_{r}-\frac{T_{l}}{J}+h i_{s q}^{*}+d_{\Omega_{r}}
$$

where $h=m \phi_{r d}$ and $d_{\Omega_{r}}=-\Delta c \Omega_{r}-\frac{T_{l}}{J}$ is the term uncertainty. Defining the speed tracking error $e_{\Omega_{r}}=\Omega_{r}-\Omega_{r}^{*}$, it follows

$$
\dot{e}_{\Omega_{r}}=-c e_{\Omega_{r}}+h i_{s q}^{*}-c \Omega_{r}^{*}-\dot{\Omega}_{r}^{*}+d_{\Omega_{r}}
$$

Define now the sliding manifold as

$$
\sigma_{\Omega_{r}}=e_{\Omega_{r}}-(k-c) \int_{0}^{t} e_{\Omega_{r}}(\tau) d \tau
$$

The Lyapunov candidate function associated to the sliding manifold (23) is defined as

$$
V_{\sigma_{\Omega_{r}}}=\frac{1}{2} \sigma_{\Omega_{r}}^{2}
$$


By computing its time derivative, one obtain

$$
\begin{aligned}
\dot{V}_{\sigma_{\Omega_{r}}} & =\sigma_{\Omega_{r}} \dot{\sigma}_{\Omega_{r}} \\
& =\sigma_{\Omega_{r}}\left[\sigma_{\Omega_{r} 1}+\sigma_{\Omega_{r} 2} i_{s q}^{*}+d_{\Omega r}\right]
\end{aligned}
$$

where $\sigma_{\Omega_{r} 1}=k e_{\Omega_{r}}+c \Omega_{r}^{*}+\dot{\Omega}_{r}^{*}$ and $\sigma_{\Omega_{r} 2}=h$.

Then, the speed controller reads

$$
i_{s q}^{*}=i_{s q, e q u}^{*}+i_{s q, n}^{*}
$$

where $i_{s q, e q u}^{*}=-\frac{\sigma_{\Omega_{r} 1}}{\sigma_{\Omega_{r} 2}}-l_{\Omega_{r}} \sigma_{\Omega_{r}}$ is the equivalent control and $i_{s q, n}^{*}=-\frac{u_{q n}}{\sigma_{\Omega_{r} 2}}$, with $u_{q n}=\eta_{\Omega_{r}} s g n\left(\sigma_{\Omega_{r}}\right)$ and

$$
\operatorname{sgn}\left(\sigma_{\Omega_{r}}\right):\left\{\begin{array}{l}
1 \text { if } \sigma_{\Omega_{r}}>0 \\
-1 \text { if } \sigma_{\Omega_{r}}<0 \\
\in[-1,1] \text { if } \sigma_{\Omega_{r}}=0
\end{array}\right.
$$

(24) becomes

$$
\dot{V}_{\sigma_{\Omega_{r}}}=-l_{\Omega_{r}} V_{\sigma_{\Omega_{r}}}+\sigma_{\Omega_{r}}\left[-\eta_{\Omega_{r}} \operatorname{sgn}\left(\sigma_{\Omega_{r}}\right)+d_{\Omega_{r}}\right]
$$

By choosing $l_{\Omega_{r}}>0$ and $\eta_{\Omega_{r}}>\max \left\{\left\|d_{\Omega_{r}}\right\|\right\}$ (defined hereafter) it follows that $\dot{V}_{\sigma_{\Omega_{r}}} \leq 0$. As $V_{\sigma_{\Omega_{r}}}$ is contracting and from assumption $(3.1-\mathrm{a}-\mathrm{b})$ then $\max \left\{\Omega_{r}\right\}:=K_{\Omega_{r}}^{\max }$ can not be greater than $\max \left\{\left|\Omega_{r}(0)\right|,\left|\Omega_{r}^{*}\right|\right\}+\left|\Delta e_{\Omega_{r}}(0)\right|$. Consequently, as $d_{\Omega_{r}}=\Delta c \Omega_{r}+\frac{T_{l}}{J}$ then $d_{\Omega_{r}}$ is bounded. Finally, $\eta_{\Omega_{r}}$ is set as $\eta_{\Omega_{r}}=\Delta c K_{\Omega_{r}}^{\max }+\frac{\varrho}{J}+b_{\Omega_{r}}$, with $b_{\Omega_{r}}$ a small positive constant. Furthermore, all the trajectories reach the sliding manifold $\sigma=0$ in a finite time and remain there. Therefore, $\sigma=0$ and equation (22) implies

$$
\dot{e}_{\Omega_{r}}=\left(k_{\Omega_{r}}-c\right) e_{\Omega_{r}}
$$

which makes that the speed tracking error $e_{\Omega_{r}}$ exponentially converges to 0 for $\left(k_{\Omega_{r}}-c\right)<0$.

Proposition 3.2 . Consider IM model (15) and assume that assumption 3.1 is satisfied. Then under the action of speed controller (25) and flux controller (19), the rotor speed and the flux track their desired trajectories.

Proof

Using

$$
V_{c}=\frac{1}{2} \sigma_{\phi_{r d}}^{2}+\frac{1}{2} \sigma_{\Omega_{r}}^{2}
$$

as a Lyapunov function candidate, then the time derivative

$$
\dot{V}_{c}=-l_{\phi_{r d}} V_{\sigma_{\phi_{r d}}}+\left[-\eta_{\phi_{r d}} \operatorname{sgn}\left(\sigma_{\phi_{r d}}\right)+\Delta a \phi_{r d}\right]-l_{\Omega_{r}} V_{\sigma_{\Omega_{r}}}+\left[-\eta_{\Omega_{r}} \operatorname{sgn}\left(\sigma_{\Omega_{r}}\right)+d_{\Omega_{r}}\right]
$$

is less than 0 .

\section{Stability analysis of the closed-loop system}

In order to implement controllers (19) and (25), the speed/flux measures are replaced by their estimates resulting in the new controllers 


$$
\begin{gathered}
i_{s d}^{*}=-l_{\hat{\phi}_{r d}} \sigma_{\hat{\phi}_{r d}}+\frac{k_{\hat{\phi}_{r d}} e_{\hat{\phi}_{r d}}+a \phi_{r d}^{*}+\dot{\phi}_{r d}^{*}-\eta_{\hat{\phi}_{r d}} \operatorname{sgn}\left(\sigma_{\hat{\phi}_{r d}}\right)}{\kappa} \\
i_{s q}^{*}=-l_{\hat{\Omega}_{r}} \sigma_{\hat{\Omega}_{r}}+\frac{k_{\hat{\Omega}_{r}} e_{\hat{\Omega}_{r}}+c \Omega_{r}^{*}+\dot{\Omega}_{r}^{*}-\eta_{\hat{\Omega}_{r}} \operatorname{sgn}\left(\sigma_{\hat{\Omega}_{r}}\right)}{m \hat{\phi}_{r d}}
\end{gathered}
$$

where

$$
\left\{\begin{aligned}
e_{\hat{\phi}_{r d}} & =\phi_{r d}^{*}-\hat{\phi}_{r d} \\
e_{\hat{\Omega}_{r}} & =\hat{\Omega}_{r}-\Omega_{r}^{*} \\
\sigma_{\hat{\phi}_{r d}} & =e_{\hat{\phi}_{r d}}+\left(k_{\phi}-a\right) \int_{0}^{t} e_{\hat{\phi}_{r d}} d \tau \\
\sigma_{\hat{\Omega}_{r}} & =e_{\hat{\Omega}_{r}}+\left(k_{\Omega_{r}}-c\right) \int_{0}^{t} e_{\hat{\Omega}_{r}} d \tau
\end{aligned}\right.
$$

The $I M$ observer must already be fluxed to ensure estimated speed tracking. In order to avoid the singularity in (28), the observer (3) is initialized with initial conditions different from zero. In practice, electrical engineers overcome this singularity by starting to track firstly the flux $\phi_{r d}$ to its reference $\phi_{r d}^{*}=$ constant . The same trick is adopted for the estimated flux $\hat{\phi}_{r d}$ by adding an offset $\varepsilon=0.05 W b$ such as

$$
i_{s q}^{*}=-l_{\hat{\Omega}_{r}} \sigma_{\hat{\Omega}_{r}}+\frac{k_{\hat{\Omega}_{r}} e_{\hat{\Omega}_{r}}+c \Omega_{r}^{*}+\dot{\Omega}_{r}^{*}-\eta_{\hat{\Omega}_{r}} \operatorname{sgn}\left(\sigma_{\hat{\Omega}_{r}}\right)}{\max \left\{\hat{\phi}_{r d}, \varepsilon\right\} m}
$$

To analyze the stability of the closed-loop system ("O-C"), the following procedure is adopted.

Argument 4.1 If it is proved that the controller (27)-(29) enables to track the estimated flux and estimated speed to their desired trajectory $\left(e=Z-X^{*} \rightarrow 0\right.$ as $\left.t \rightarrow \infty\right)$, then, it is ensured that the $I M$ flux and the speed practically converge to their desired trajectories.

-Estimated flux tracking. Define the candidate Lyapunov function $V_{\sigma_{\hat{\phi}_{r d}}}=\frac{1}{2} \sigma_{\hat{\phi}_{r d}}^{2}$, which time derivative is $\dot{V}_{\hat{\phi}_{r d}}=-l_{\hat{\phi}_{r d}} V_{\sigma_{\hat{\phi}_{r d}}}-\sigma_{\hat{\phi}_{r d}}\left[\eta_{\hat{\phi}_{r d}} \operatorname{sgn}\left(\sigma_{\hat{\phi}_{r d}}\right)+\mathbf{M} g_{\hat{\phi}_{r d}} \epsilon_{i_{s \beta}}\right]$, where $g_{\hat{\phi}_{r d}}$ is the gain of the estimated flux $\hat{\phi}_{r d}$. By choosing $l_{\hat{\phi}_{r d}}>0$ and $\eta_{\hat{\phi}_{r d}}>\max \left\{\mathbf{M}\left\|g_{\hat{\phi}_{r d}}\right\|\left\|\epsilon_{i_{s \beta}}\right\|\right\}$ (defined hereafter) it follows that $V_{\sigma_{\hat{\phi}_{r d}}} \leq 0$. Now, imposing the initial states of the observer to be in $\mathcal{D}$ and as $V_{\sigma_{\hat{\phi}_{r d}}}$ is contracting, then from assumption (3.1-b), $\max \left\{\hat{\phi}_{r d}\right\}:=K_{\hat{\phi}_{r d}}^{\max }$ can not be greater than $\max \left\{\hat{\phi}_{r d}(0), \phi_{r d}^{*}\right\}+\left|\Delta e_{\hat{\phi}_{r d}}(0)\right|$. From where it can be deduced that $\left\|g_{\hat{\phi}_{r d}}\right\|$ is bounded. Moreover, as $V_{0}=V_{1}+V_{2}$ (see the proposition 2.4 proof) is practically stable and does not depend on $u$, then $\left\|\epsilon_{i_{s \beta}}\right\|$ is bounded. Finally, $\eta_{\hat{\phi}_{r d}}$ is set as

$$
\eta_{\hat{\phi}_{r d}}=K_{g_{\hat{\phi}_{r d}}}^{\max } K_{\epsilon_{i_{s}}}^{\max }+c_{1}, c_{1}>0
$$

-Estimated speed tracking. Consider the candidate Lyapunov function $V_{\hat{\Omega}_{r}}=\frac{1}{2} \hat{\Omega}_{r}^{2}$, which time derivative is $\dot{V}_{\sigma_{\hat{\Omega}_{r}}}=-l_{\hat{\Omega}_{r}} V_{\hat{\Omega}_{r}}-\sigma_{\hat{\phi}_{r d}}\left[\eta_{\hat{\Omega}_{r}} \operatorname{sgn}\left(\sigma_{\hat{\Omega}_{r}}\right)+\mathbf{M} g_{\hat{\Omega}_{r}} \epsilon_{i_{s \alpha}}\right]$, where $g_{\hat{\Omega}_{r}}$ is the gain of the estimated speed $\hat{\Omega}_{r}$.

By choosing $l_{\hat{\Omega}_{r}}>0$ and $\eta_{\hat{\Omega}_{r}}>\max \left\{\mathbf{M}\left\|g_{\hat{\Omega}_{r}}\right\|\left\|\epsilon_{i_{s \alpha}}\right\|\right\}$ it follows that $V_{\hat{\Omega}_{r}} \leq 0$. Following the same ideas as above leads to

$$
\eta_{\hat{\Omega}_{r}}=K_{g_{\hat{\Omega}_{r}}}^{\max } K_{\epsilon_{i_{s \alpha}}}^{\max }+c_{2}, c_{2}>0
$$


Lemma 4.2 Consider observer (3) initialized in $\mathcal{D}$ and assume that assumption (3.1-a-b) holds. Then, under the action of the controller (27)-(29), the rotor flux and rotor speed estimations track their desired trajectories.

Proof Using

$$
V_{c}=\frac{1}{2} \sigma_{\hat{\phi}_{r d}}^{2}+\frac{1}{2} \sigma_{\hat{\Omega}_{r}}^{2}
$$

as a Lyapunov function candidate, it follows

$$
\dot{V}_{c}=-l_{\hat{\phi}_{r d}} V_{\sigma_{\hat{\phi}_{r d}}}-\sigma_{\hat{\phi}_{r d}}\left[\eta_{\hat{\phi}_{r d}} \operatorname{sgn}\left(\sigma_{\hat{\phi}_{r d}}\right)+\mathbf{M} g_{\hat{\phi}_{r d}} \epsilon_{i_{s \beta}}\right]-l_{\hat{\Omega}_{r}} V_{\sigma_{\hat{\Omega}_{r}}}-\sigma_{\hat{\Omega}_{r}}\left[\eta_{\hat{\Omega}_{r}} \operatorname{sgn}\left(\sigma_{\hat{\Omega}_{r}}\right)+\mathbf{M} g_{\hat{\Omega}_{r}} \epsilon_{i_{s \alpha}}\right] \leq 0 .
$$

Now, a practical stability result of the proposed observer based control for the $I M$ is given.

TheOREM 4.3 Consider the controller (27)-(29) and property (2.2). If the observer (3) is initialized on $\mathcal{D}$ and assuming the assumption (3.1) is satisfied, then

- The IM estimated state practically converges to the real state.

- The estimated flux and speed exponentially converge to their desired trajectories.

- Finally, the real flux and speed of IM converge practically to their desired trajectories.

Proof The proof follows from proposition (2.4), lemma (4.2) and argument (4.1).

\section{EXPERIMENTAL RESULTS}

\subsection{Control Benchmark}

A Sensorless Control Benchmark defines the adequate reference trajectories to evaluate the performances of the sensorless control algorithms under the following operating conditions (Figure 3).

Area 1. Low speed with nominal load (from 1 to $3 \mathrm{~s}$ ).

Area 2. High speed with nominal load (from $4 \mathrm{~s}$ to $6 \mathrm{~s}$ ).

Area 3. Very low speed (zero frequency) with nominal load (the IM is unobservable from $7 s$ to $9 s$ ).
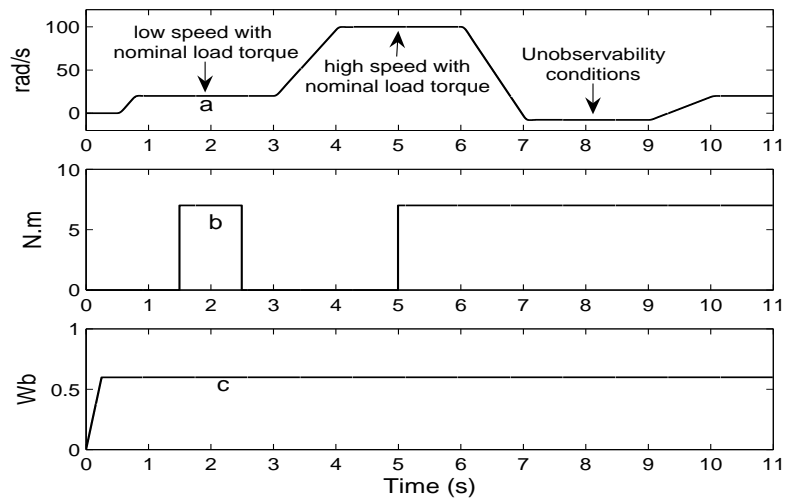

${ }_{1}$ see section 2.2 for more details 
Figure 3. Control benchmark trajectories: a- Reference speed: $\Omega^{*}(\mathrm{rad} / \mathrm{s}), \mathrm{b}-$ Reference load: $T_{l}^{*}(\mathrm{~N} . \mathrm{m})$, c-Reference flux: $\phi_{r d}^{*}(\mathrm{~Wb})$.

\section{$5.2 \quad$ Experimental results}

Here, the experimental results obtained with the proposed controller using the observer are given. The tests have been performed with the following induction motor values:

\begin{tabular}{|c|c||c|c|}
\hline Nominal rate power & $1.5 \mathrm{~kW}$ & $R_{s}$ & $1.47 \Omega$ \\
\hline Nominal angular speed & $1430 \mathrm{rpm}$ & $R_{r}$ & $0.79 \Omega$ \\
\hline Number of pole pairs & 2 & $L_{s}$ & $0.105 \mathrm{H}$ \\
\hline Nominal voltage & $220 \mathrm{~V}$ & $L_{r}$ & $0.094 \mathrm{H}$ \\
\hline Nominal current & $6.1 \mathrm{~A}$ & $J, f_{v}$ & $0.0077 \mathrm{Kg}^{2} \mathrm{~m}^{2}, 0.0029 \mathrm{Nm} / \mathrm{rad} / \mathrm{s}$ \\
\hline
\end{tabular}

and the guidelines parameters tuning for the observer and the controller are given as follows :

- For the observer given by (3), $\theta_{1}$ and $\theta_{1}$ are chosen to satisfy (11). From (11), it is easy to see that $\theta_{1}>N v$ and $\theta_{2}>\frac{N}{v}$ where $\left.\left.v \in\right] 01\right]$. So, $\theta_{1}$ is proportional to $v$ while $\theta_{2}$ is inversely proportional. We choose $\theta_{1}=1$ and $\theta_{2}=5000$.

- For the controller given by (14), the parameters $K p_{v d}, K p_{v q}, K_{I v d}, K_{I v q}$ are determined as follows :

Considering the dynamic equations of $i_{s d}$ and $i_{s q}$ given by (13) without nonlinearities and coupling terms

$$
\left\{\begin{array}{l}
\dot{i}_{s d}=-\gamma i_{s d}+m_{1} u_{s d} \\
\dot{i}_{s q}=-\gamma i_{s q}+m_{1} u_{s q}
\end{array}\right.
$$

Writing the transfer function which lies the stator currents of (32) with their references given by (14) as a second order system in closed loop, it follows

$$
\left\{\begin{array}{l}
\frac{i_{s d}}{i_{s d}^{*}}=\frac{w_{n d}^{2}}{s^{2}+2 \zeta w_{n d}+w_{n d}^{2}} \\
\frac{i_{s q}}{i_{s q}^{*}}=\frac{w_{n q}^{2}}{s^{2}+2 \zeta w_{n q}+w_{n q}^{2}}
\end{array}\right.
$$

By imposing $\zeta=1$ to avoid peaking and a currents bands-widths $F_{B D}$ at least less than a middle of $F_{e}=1 / T_{e}$ where $T_{e}=200 \mu s$ is the sampling time:

$$
\left\{\begin{aligned}
\zeta & =1 \\
w_{n d} & =2 \pi F_{B D} \\
w_{n q} & =2 \pi F_{B D}
\end{aligned}\right.
$$

the parameters $K p_{v d}, K p_{v q}, K_{I v d}, K_{I v q}$ can be established:

$$
\left\{\begin{array}{l}
K p_{v d}=\frac{2 \zeta-\gamma}{m_{1}}, \quad T i_{v d}=\frac{2 \zeta-\gamma}{w_{n d}^{2}} \\
K p_{v q}=\frac{2 \zeta-\gamma}{m_{1}}, \quad T i_{v q}=\frac{2 \zeta-\gamma}{w_{n q}^{2}}
\end{array}\right.
$$

where $K_{I v d}=\frac{K p_{v d}}{T i_{v d}}$ and $K_{I v q}=\frac{K p_{v q}}{T i_{v q}}$. We choose $K p_{v d}=2, K p_{v q}=2, K_{I v d}=0.05, K_{I v q}=0.05$. 
- For the controller given by (27)-(29), $l_{\hat{\phi}_{r d}}>0, l_{\hat{\Omega}_{r}}>0$ and the parameters $k_{\hat{\phi}_{r d}}, k_{\hat{\Omega}_{r}}, \eta_{\hat{\phi}_{r d}}$ and $\eta_{\hat{\Omega}_{r}}$ are chosen to satisfy respectively (20), (26), (30), (31). We choose $\eta_{\hat{\phi}_{r d}}=10, k_{\hat{\phi}_{r d}}=-80, l_{\hat{\phi}_{r d}}=4, \eta_{\hat{\Omega}_{r}}=5$, $k_{\hat{\Omega}_{r}}=-40, l_{\hat{\Omega}_{r}}=2$.

The block diagram scheme used in experimental set-up to test the law control with observer is presented in figure 4 . The block "Intercon. observers" is constituted by the two interconnected observers we have designed. This block uses only the current and stator measurements in the reference fixed frame $(\alpha-\beta)$ to estimate the speed, the flux amplitude and the flux angle. The block "Sliding and Field Oriented Control" contains the proposed controller. This block uses the estimates of speed, flux amplitude and flux angle given by the block "Intercon. observers" and the current measurements after using the transformation of Park and Concordia. Then, it gives the inputs control in the reference fixed frame $(\mathrm{a}, \mathrm{b}, \mathrm{c})$ after using the inverse transformations of Park and Concordia. These control inputs drive the inverter to impose the speed and flux reference trajectories (defined by the "Control Benchmark"). The track of the reference load torque trajectory (also defined in the "Control Benchmark") is imposed by the connected synchronous motor.

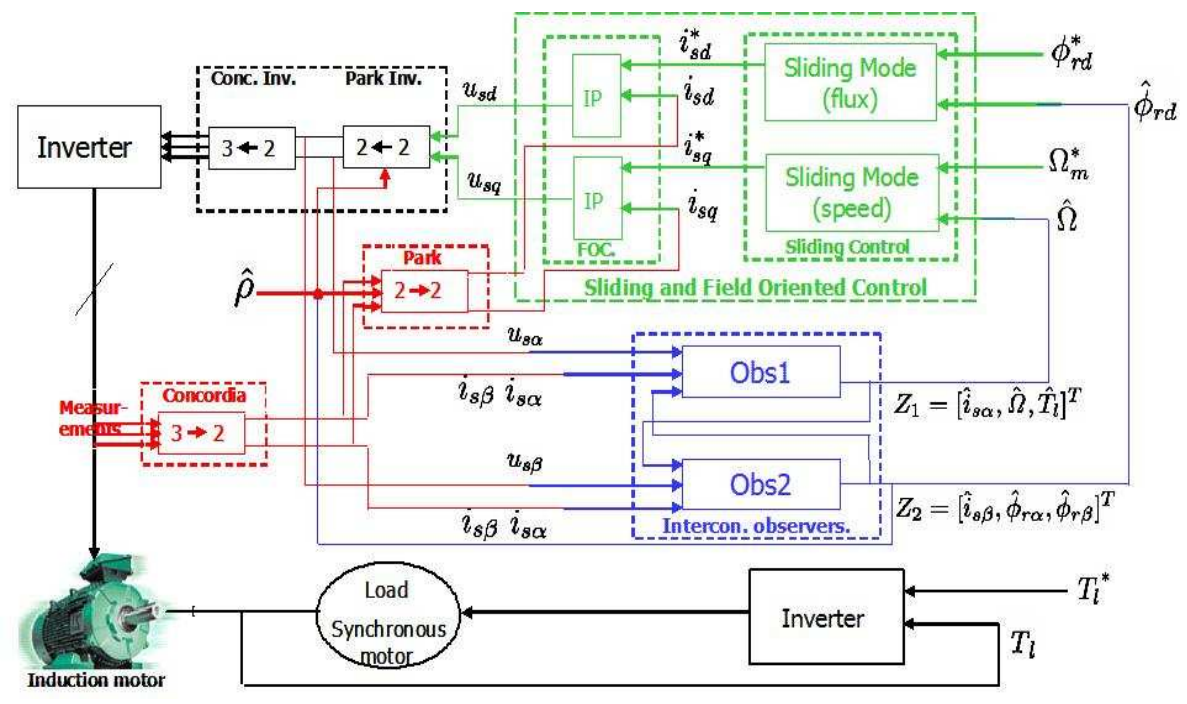

Figure 4. Diagram of the controller-observer scheme.

5.2.1 Case with identified parameters. Figures 5 and 6 show the experimental results in case where identified parameters are used to design the flux and speed sliding mode control with interconnected observers (Controller-Observer Scheme).

Remark 1 Due to experimental conditions (temperature, ...), the identified parameters are not exactly the real parameters of induction motor. The control experimental conditions are nearly different compared to the identification conditions and moreover the identification methodology has a certain uncertainty in its results. Thus this case is already a first robustness test.

We can remark that both systems "Control+Observer" give good performances i) in term of trajectory tracking: the motor speed $\left(\Omega_{r}\right.$ in figure 5$)$ tracks correctly its reference ( $\Omega_{r}^{*}$ in figure 5$)$ even under unobservable conditions (between 7 and $9 \mathrm{sec}$ ), nevertheless it appears a small static error when the motor is under unobservable conditions, ii) in term of perturbation rejection: the load torque is well rejected under low speed and high speed. Nevertheless it appears a small static error when the load is applied (Figure 5: $1.5 \mathrm{~s}$ ) and when it is removed (Figure 5: $2.5 \mathrm{sec})$. For estimated flux $\left(\hat{\phi}_{r d}\right.$ in figure 6$)$, the same conclusion is given and moreover it exists a small peaking at the beginning (Figure 6) which is due to initial conditions. Load torque estimate is plotted in figure 9. The determinant $\mathbf{D}$ and the switch function $\mathbf{M}$ introduced respectively by definition (2.3) and observer (3) are shown in figure 10 with $D_{\min }=0.05$. The control efforts $V_{s d}$ and $V_{s q}$ are shown in figure 7 . In figure 8 the measured and reference currents of $i_{s d}$ and $i_{s q}$ are displayed. 


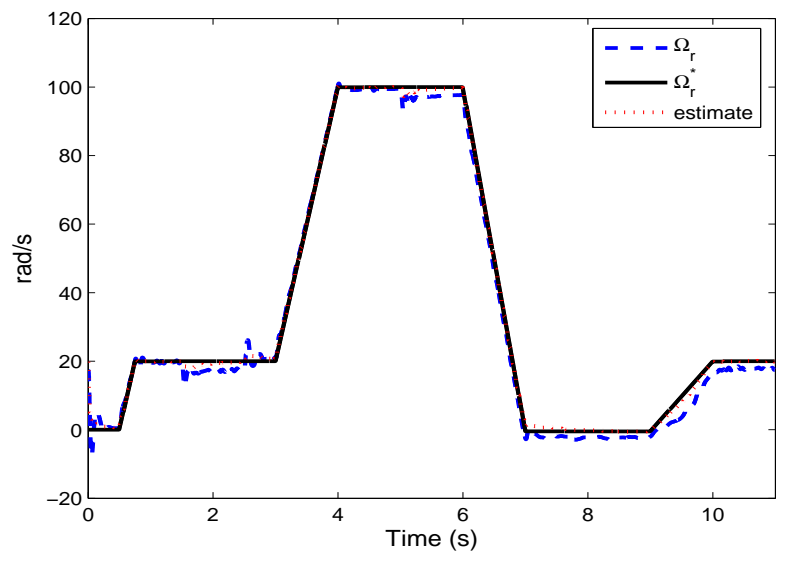

Figure 5. Reference speed $\Omega_{r}^{*}$, motor speed $\Omega_{r}$, estimated speed $\hat{\Omega}_{r}(\mathrm{rad} / \mathrm{s})$

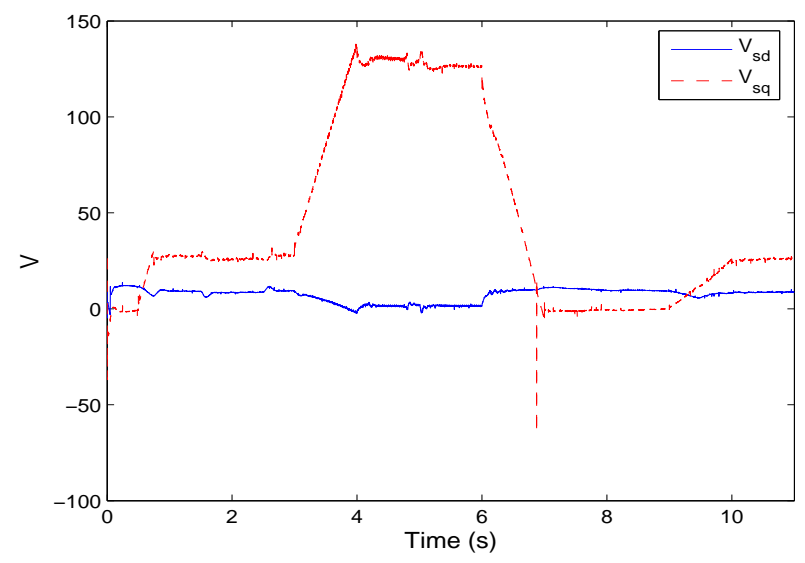

Figure 7. $V_{s d}$ and $V_{s q}(\mathrm{~V})$

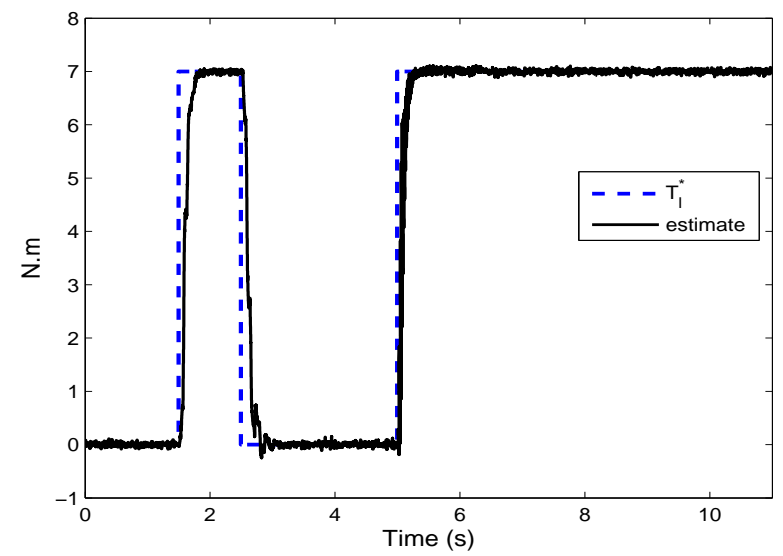

Figure 9. $T_{l}^{*}$ and $\hat{T}_{l}$ (N.m)

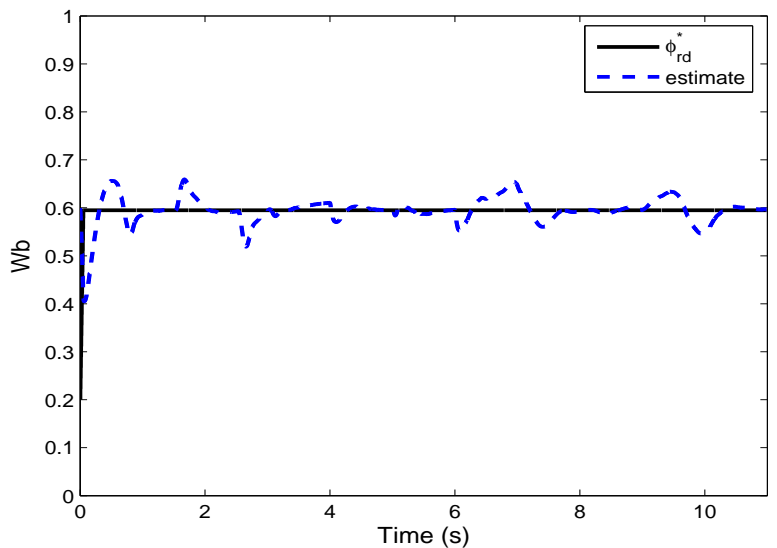

Figure 6. Flux, (Wb)
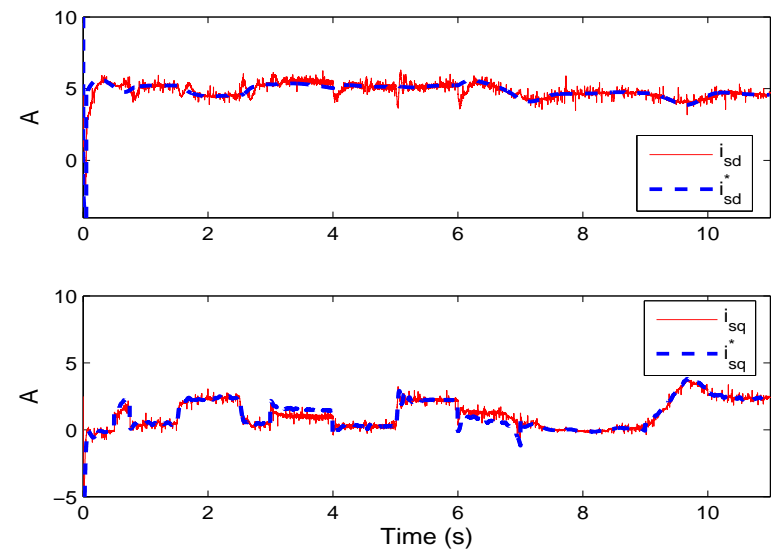

Figure 8. $i_{s d}$ and $i_{s q},(\mathrm{~A})$

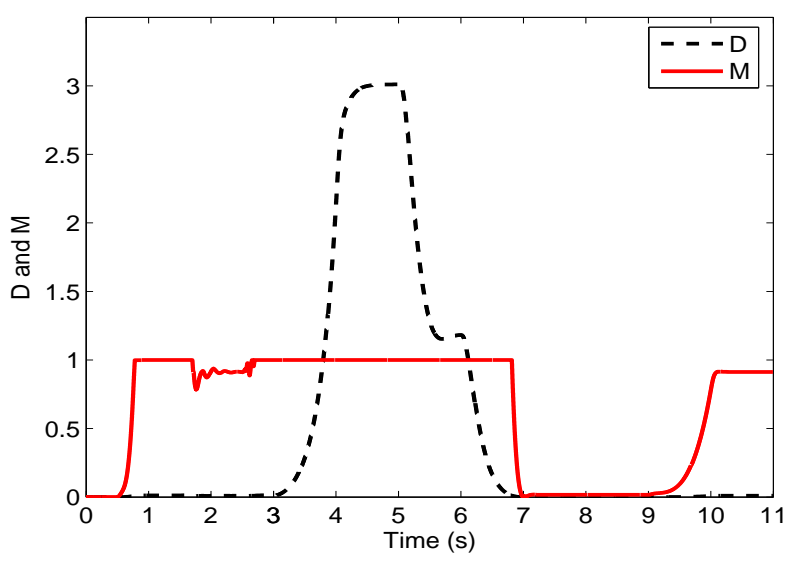

Figure 10. D and M

Case 1: experimental results with identified parameters.

5.2.2 Robustness cases. The interest now is to check the robustness of the designed Control-Observer with respect to motor parameters variation. We have considered two robustness cases : a stator resistance variation of $+50 \%$ and a rotor resistance variation of $+50 \%$ with respect to the values of the previous case.

Case with $+\mathbf{5 0 \%}$ of $R_{r}$ : the results that we have obtained are depicted in figures 11 and 12 . 
Compared with case 1 , the motor speed $\left(\Omega_{r}\right.$ in figure 11) is more affected by the increase of the rotor resistance at high speed when the load torque is applied (Figure 11: $5 \mathrm{~s}$ ). But the speed tracking when the motor is under unobservable conditions remains same (Figure 11: from 7 to $9 \mathrm{~s}$ ). For estimated flux (Figure 12), the results are nearly similar compared with the case 1 . The control efforts $V_{s d}$ and $V_{s q}$ are shown in figure 13. In figure 14 the measured and reference currents of $i_{s d}$ and $i_{s q}$ are displayed. The load torque estimate is not depicted because it is nearly similar to the one of case 1.

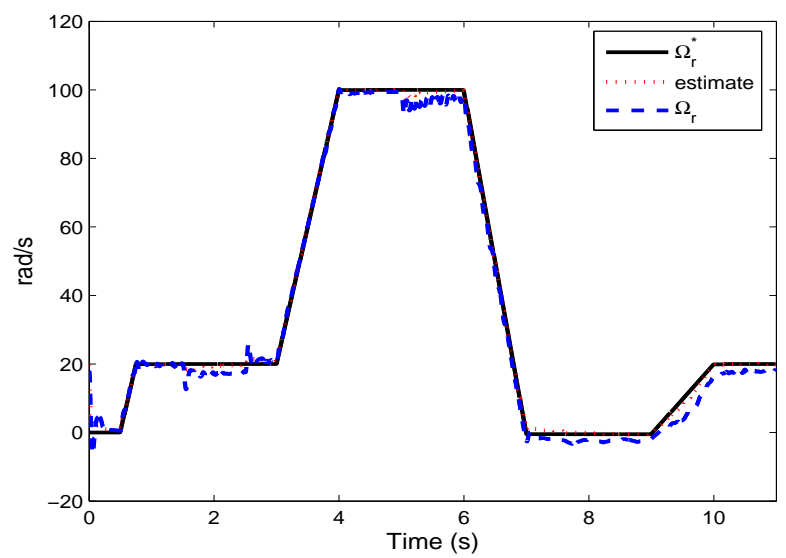

Figure 11. Reference speed $\Omega_{r}^{*}$, motor speed $\Omega_{r}$, estimated speed $\hat{\Omega}_{r}(\mathrm{rad} / \mathrm{s})$

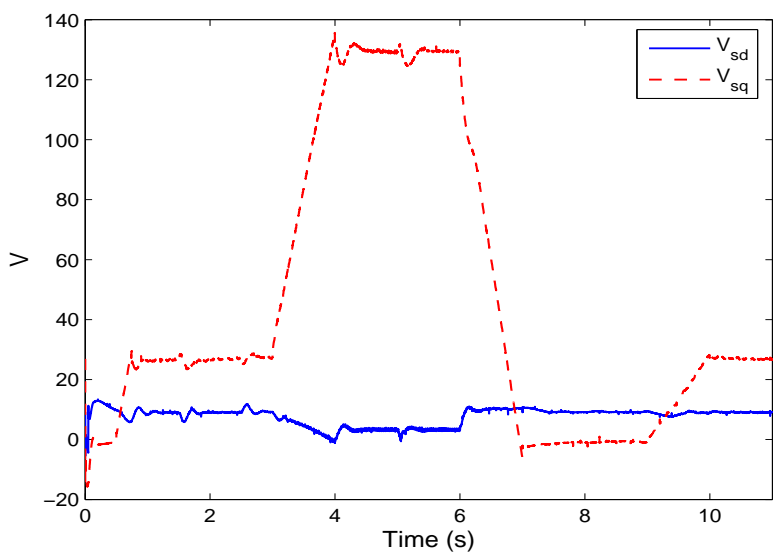

Figure 13. $V_{s d}$ and $V_{s q}(\mathrm{~V})$

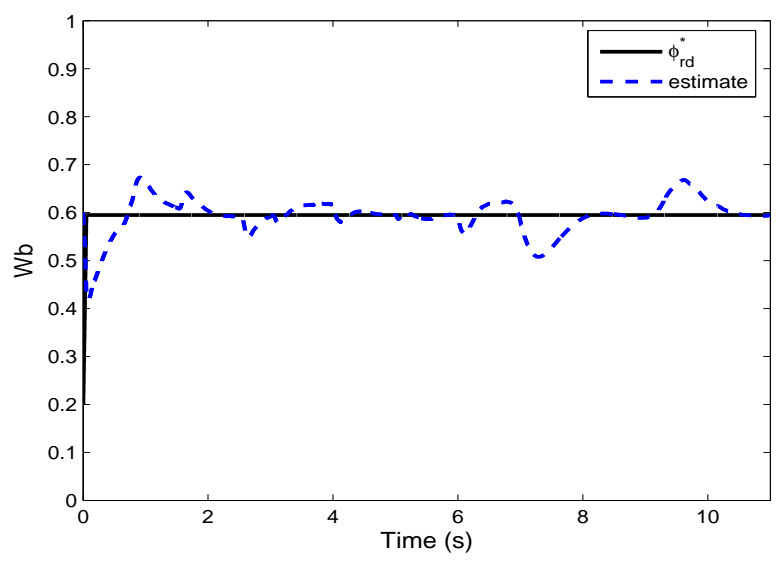

Figure 12. Flux, (rad/s)
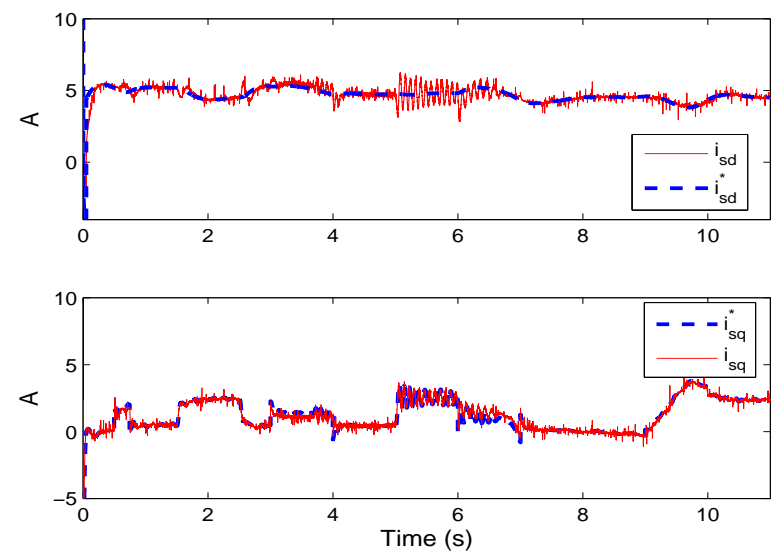

Figure 14. $i_{s d}$ and $i_{s q},(\mathrm{~A})$

Case 2: experimental results with rotor resistance variation $(+50 \%)$.

Case with $+\mathbf{5 0 \%}$ of $R_{s}$ : in figures 15 and 16 , the sensitivity of both "Control + Observer" with respect to the variation of the stator resistance is showed. The performances of both "Control+Observer" are nearly similar with the case 1 for the speed motor (Figure 15. b) and estimated flux (Figure 16. b). However, an improvement can be remarked for motor speed at high speed when the load torque is applied (Figure 11: 5 s): the oscillations are removed, it remains only a small static error. This improvement can be explained by the fact that the parameters are close to the real parameters of induction motor as it can be obtained by simulation with the same parameters for model and both "Control+Observer". The control efforts $V_{s d}$ and $V_{s q}$ are shown in figure 17. In figure 18 the measured and reference currents of $i_{s d}$ and $i_{s q}$ are displayed. The load torque estimate is not depicted because it is nearly similar compared to case 1 . 


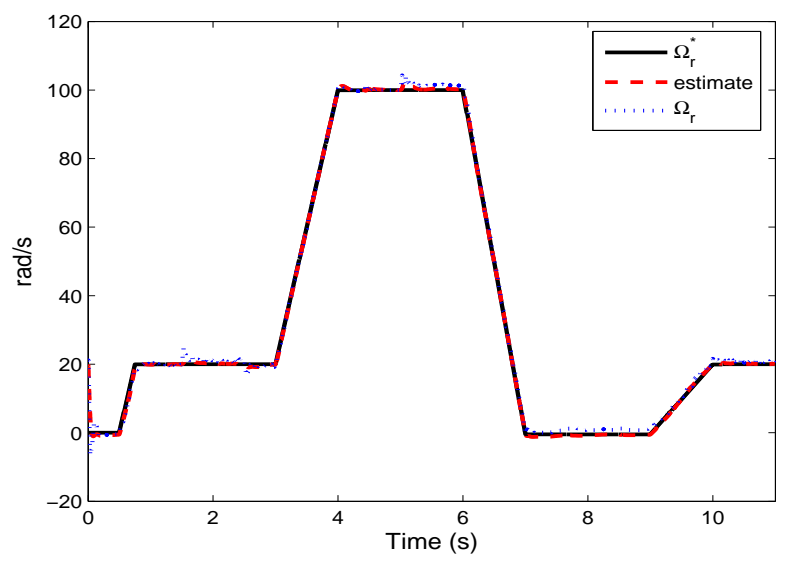

Figure 15. Reference speed $\Omega_{r}^{*}$, motor speed $\Omega_{r}$, estimated speed $\hat{\Omega}_{r}(\mathrm{rad} / \mathrm{s})$

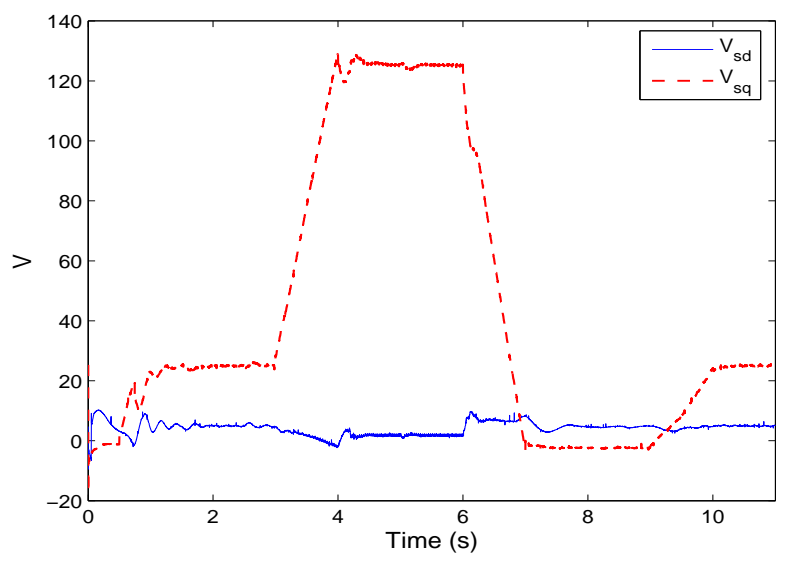

Figure 17. $V_{s d}$ and $V_{s q}(\mathrm{~V})$

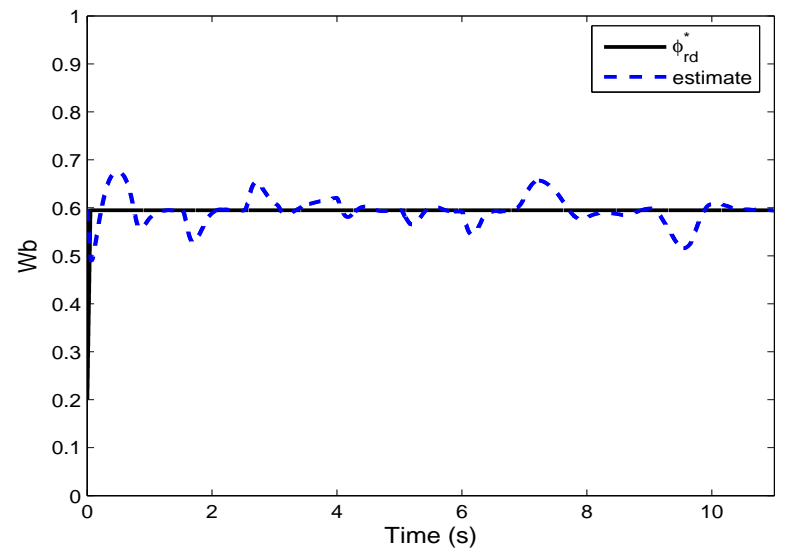

Figure 16. Flux, (rad/s)
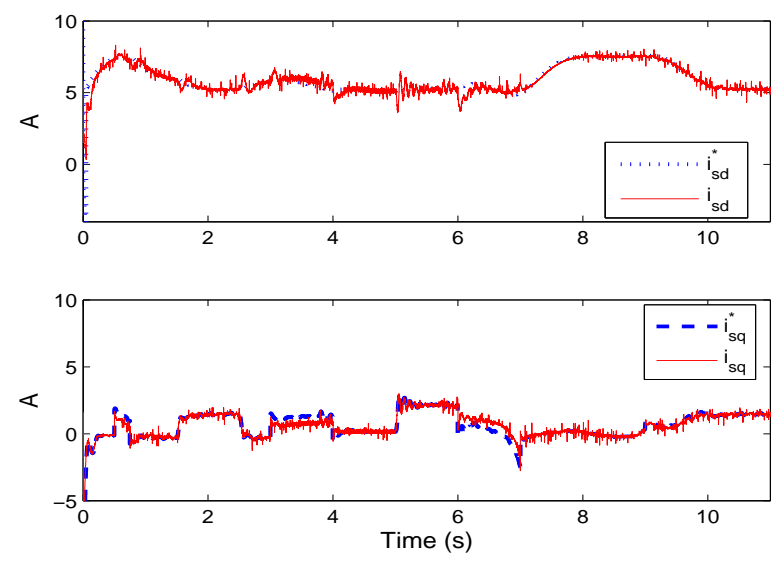

Figure 18. $i_{s d}$ and $i_{s q},(\mathrm{~A})$

Case 3: experimental results with stator resistance variation $(+50 \%)$.

\section{CONCLUSION}

In this paper, a SIM observer has firstly been designed. This later structurally takes into account the unobservable conditions of the $I M$. The gains are chosen according to the ratio between the unobservable and observable time intervals. Secondly, the estimated speed and flux are used to design a FOC-SMC in order to steer the estimated speed and flux magnitude to their desired trajectories in a finite time. Consequently, the performance of the observer based control scheme "O-C" scheme is related to the observer behavior, which is a natural consequence of the unobservable ${ }^{1}$ sequences. Finally, this scheme has been tested and validated on an experimental set-up using the reference trajectories of a realistic proposed benchmark where the $I M$ remains in the unobservable sequences and not just go across.

\section{References}

A.N. Atassi, Khalil, H.K., "A separation principle for the stabilization of a class of nonlinear systems", IEEE Trans. Automat. Contr., vol. 44, pp. 1672-1687, 1999.

\footnotetext{
${ }^{1}$ see section (2.2) for more details
} 
A. Balluchi, M.D. Di Benedetto, L. Benvenuti, A. L. Sangiovanni-Vincentelli, "Observability for Hybrid Systems", IEEE Conf. Decision. Contr., Maui, Hawaii, 2003.

G. Besançon and H. Hammouri, "On Observer Design for Interconnected Systems", Journal of Mathematical Systems, Estimation and Control, 8, 4, 1998.

G. Besançon, "A viewpoint on Observability and Observer Design for Nonlinear Systems", New Directions in Nonlinear Observer Design, Lecture Notes in Control and information Sciences, Springer, No.244, 1999.

F. Blaschke, "The principle of field orientation applied to the new transvector closed-loop control system for rotating field machines", Siemens-Rev., 39, pp. 217-220, 1972.

C. Canudas de Wit, A. Youssef, J.P. Barbot, Ph. Martin and F. Malrait, "Observability Conditions of Induction Motors at low frequencies", IEEE Conference on Decision and Control, Sydney, Australia, 2000.

J. Chiasson, Modeling and High-Performance Control of Electric Machines", IEEE Series on Power Engineering, Wiley-Interscience, 2005.

M. Feemster, P. Aquino, D. M. Dawson and A. Behal, "Sensorless rotor velocity tracking control for induction motors", IEEE Transactions on Control Systems Technology, 9, 4, pp. 645-653, 2001.

M. Ghanes, J. DeLeon, and A. Glumineau, "Observability Study and Observer-Based Interconnected Form for Sensorless Induction Motor", IEEE Conference on Decision and Control CDC, San Diego, California, USA, 2006.

J. Holtz, "Sensorless control of induction machines-with or without signal injection" IEEE Trans. Ind. Electron., 53, 1, pp. 7-30, 2006.

S. Ibarra-Rojas, J. Moreno and G. Espinosa, "Global observability analysis of sensorless induction motor", Automatica, Vol. 40, Issue 6, pp. 1079-1085, 2004.

M. Jankovic, "Adaptative Nonlinear Output Feedback Tracking with a Partial High-Gain Observer Backstepping", IEEE Trans. Automat. Contr., 42, 1, pp. 106-113, 1997.

H. Kubota and K. Matsuse, "Speed sensorless filed-oriented control of induction motor with rotor resistance adaptation" IEEE Transactions on Industry Applications, 30, 5, pp.344-348, Mar./Apr., 1994.

Laskhmikanthan, V., Leila, S. and Martynyuk, A.A., "Practical stability of nonlinear systems". Word Scientific. ISBN 978-9810203566. 1990.

Y. C. Lin, Fu, L. C., and Tsai, C. Y., "Non-linear sensorless indirect adaptive speed control of induction motor with unknown rotor resistance andload", International Journal of Adaptive Control and Signal Processing, 14, pp. 109-140, 2000.

R. Marino, P. Tomei and C. M. Verrelli, "A nonlinear tracking control for sensorless induction motors "Automatica, 41, 6, pp. 1071-1077, 2005.

R. Marino, P. Tomei and C. M. Verrelli, "A nonlinear tracking control for sensorless induction motors with uncertain load torque" Inter. J. of Adaptive Contr. and Signal Processing, March, 2007.

M. Montanari, S. Peresada, A. Tilli, "A speed-sensorless indirect field-oriented control for induction motors based on high gain speed estimation", Automatica Vol. 42, Issue 10, pp. 1637-1650, 2006.

S. Peresada, A. Tonielli and R. Morici (1999). High performance indirect field oriented output feedback control of induction motors, Automatica Vol. 35, Issue 6, pp. 1033-1047, 1999.

E. Panteley and A. Loria, "On global uniform asymptotic stability of cascade time-varying systems in cascasde", Systems and Control Letters, 33, pp.131-138, 1998.

H. Tajima and Y. Hori, "Speedsensorless field-orientation control of the induction machine", IEEE Transactions on Industry Applications, 29, 1, pp. 175-180, 1993.

Utkin, V.I. "Sliding Modes in Control Optimization". Springer-Verlag. ISBN 9780387535166. (1992).

I. Zein, "Extended Filter Kalman and Luenberger Observer Application to Control of Induction Motor", Phd thesis, Université de Technologie de Compiegne, septembre 2000. 


\section{Annexe: Positiveness proof of $S_{i}(t)$}

Consider the expressions of $\dot{S}_{i}, i=1,2$ given by observer (3):

$$
\dot{S}_{i}=\mathbf{M}\left(-\theta_{i} S_{i}-A_{i}^{T}\left(Z_{j}\right) S_{i}-S_{i} A_{i}\left(Z_{j}\right)+C_{i}^{T} C_{i}\right),
$$

with $j=2$ if $i=1$ and $j=1$ if $i=2$.

We denote by $\Phi_{i}(t, r)$ the unique solution of:

$$
\dot{\Phi}_{i}(t, r)=\frac{d \Phi_{i}(t, r)}{d r}=A_{i}\left(Z_{j}\right) \Phi_{i}(t, r)
$$

such that

$$
\Phi_{i}(t, t)=I
$$

where $I$ is the identity matrix and $r$ vary from 0 to $t$.

Two cases are considered:

$M=1$ : observable conditions where $r$ vary from 0 to $t_{1}$.

$M=0$ : unobservable conditions where $r$ vary from $t_{1}$ to $t$.

1) Case where $M=1$ (observable conditions) $r \in\left[0, t_{1}\right]$ :

Multiplying each term of $(33)$ by $\Phi_{i}\left(t_{1}, r\right)^{T}$ in left side and by $\Phi_{i}\left(t_{1}, r\right)$ in right side, we get

$$
\left(\begin{array}{c}
\Phi_{i}\left(t_{1}, r\right)^{T} \dot{S}_{i}(r) \Phi_{i}\left(t_{1}, r\right)+\theta_{i} \Phi_{i}\left(t_{1}, r\right)^{T} S_{i}(r) \Phi_{i}\left(t_{1}, r\right) \\
+\Phi_{i}\left(t_{1}, r\right)^{T} A_{i}^{T}\left(Z_{j}\right) S_{i}(r) \Phi_{i}\left(t_{1}, r\right) \\
+\Phi_{i}\left(t_{1}, r\right)^{T} S_{i}(r) A_{i}\left(Z_{j}\right) \Phi_{i}\left(t_{1}, r\right)
\end{array}\right)=\Phi_{i}\left(t_{1}, r\right)^{T} C_{i}^{T} C_{i} \Phi_{i}\left(t_{1}, r\right)
$$

By multiplying both sides of $(36)$ by $\exp ^{\theta_{i} r}$ it follows:

$$
\left(\begin{array}{c}
\exp ^{\theta_{i} r} \Phi_{i}\left(t_{1}, r\right)^{T} \dot{S}_{i}(r) \Phi_{i}\left(t_{1}, r\right) \\
+\theta_{i} \exp ^{\theta_{i} r} \Phi_{i}\left(t_{1}, r\right)^{T} S_{i}(r) \Phi_{i}\left(t_{1}, r\right) \\
+\exp ^{\theta_{i} r} \Phi_{i}\left(t_{1}, r\right)^{T} A_{i}^{T}\left(Z_{j}\right) S_{i}(r) \Phi_{i}\left(t_{1}, r\right) \\
+\exp ^{\theta_{i} r} \Phi_{i}\left(t_{1}, r\right)^{T} S_{i}(r) A_{i}\left(Z_{j}\right) \Phi_{i}\left(t_{1}, r\right)
\end{array}\right)=\exp ^{\theta_{i} r} \Phi_{i}\left(t_{1}, r\right)^{T} C_{i}^{T} C_{i} \Phi_{i}\left(t_{1}, r\right)
$$

It can be remarked that the left side of (37) is the derivative of $\exp ^{\theta_{i} r} \Phi_{i}\left(t_{1}, r\right)^{T} S_{i}(r) \Phi_{i}\left(t_{1}, r\right)$ with respect to $r$. Then we can write:

$$
\overbrace{\left(\exp ^{\theta_{i} r} \Phi_{i}\left(t_{1}, r\right)^{T} S_{i}(r) \Phi_{i}\left(t_{1}, r\right)\right)}^{i}=\exp ^{\theta_{i} r} \Phi_{i}\left(t_{1}, r\right)^{T} C_{i}^{T} C_{i} \Phi_{i}\left(t_{1}, r\right)
$$

The solution $S_{i}\left(t_{1}\right)$ is obtained by integrating the equation (38) with $r$ vary from 0 to $t_{1}$ as 


$$
\int_{0}^{t_{1}} \overbrace{\left(\exp ^{\theta_{i} r} \Phi_{i}\left(t_{1}, r\right)^{T} S_{i}(r) \Phi_{i}\left(t_{1}, r\right)\right)} d r=\int_{0}^{t_{1}} \exp ^{\theta_{i} r} \Phi_{i}\left(t_{1}, r\right)^{T} C_{i}^{T} C_{i} \Phi_{i}\left(t_{1}, r\right) d r
$$

Then, equation (39) gives

$$
\exp ^{\theta_{i} t_{1}} \Phi_{i}\left(t_{1}, t_{1}\right)^{T} S_{i}\left(t_{1}\right) \Phi_{i}\left(t_{1}, t_{1}\right)-\Phi_{i}\left(t_{1}, 0\right)^{T} S_{i}(0) \Phi_{i}\left(t_{1}, 0\right)=\int_{0}^{t_{1}} \exp ^{\theta_{i} r} \Phi_{i}\left(t_{1}, r\right)^{T} C_{i}^{T} C_{i} \Phi_{i}\left(t_{1}, r\right) d r
$$

Finally, by using the property (35) in equation (40), the solution $S_{i}\left(t_{1}\right)$ yields

$$
S_{i}\left(t_{1}\right)=\exp ^{-\theta_{i} t_{1}} \Phi_{i}\left(t_{1}, 0\right)^{T} S_{i}(0) \Phi_{i}\left(t_{1}, 0\right)+\int_{0}^{t} \exp ^{-\theta_{i}\left(t_{1}-r\right)} \Phi_{i}\left(t_{1}, r\right)^{T} C_{i}^{T} C_{i} \Phi_{i}\left(t_{1}, r\right) d r
$$

From (41), we can remark that $S_{i}\left(t_{1}\right)$ is definite positive with $S_{i}(0)>0$ due to the fact that $Z_{1}$ and $Z_{2}$ of equations (34) are regularly persistent (see remark 1.2). Then the positiveness of $S_{i}\left(t_{1}\right)$ is demonstrated.

2) Case where $\mathrm{M}=0$ (unobservable conditions) $r \in\left[t_{1}, t\right]$ :

Equation (33) becomes:

$$
\dot{S}_{i}(r)=0
$$

By integrating equation (42) with $r$ varying from $t_{1}$ to $t$, we get

$$
S_{i}(t)=S_{i}\left(t_{1}\right)
$$

It can be noted that the final condition $S_{i}\left(t_{1}\right)$ of the interval time where $I M$ is observable is equal to the final condition $S_{i}(t)$ of the interval time where IM is unobservable since $S_{i}$ remains constant during this unobservable interval of time. Then $S_{i}(t)$ (equation 43) is positive definite.

This ends the proof. 